\title{
SENSITIVITY ANALYSIS OF A NONLINEAR OBSTACLE PLATE PROBLEM*
}

\author{
Isabel N. Figueiredo ${ }^{1}$ And Carlos F. Leal ${ }^{1,2}$
}

\begin{abstract}
We analyse the sensitivity of the solution of a nonlinear obstacle plate problem, with respect to small perturbations of the middle plane of the plate. This analysis, which generalizes the results of $[9,10]$ for the linear case, is done by application of an abstract variational result [6], where the sensitivity of parameterized variational inequalities in Banach spaces, without uniqueness of solution, is quantified in terms of a generalized derivative, that is the proto-derivative. We prove that the hypotheses required by this abstract sensitivity result are verified for the nonlinear obstacle plate problem. Namely, the constraint set defined by the obstacle is polyhedric and the mapping involved in the definition of the plate problem, considered as a function of the middle plane of the plate, is semi-differentiable. The verification of these two conditions enable to conclude that the sensitivity is characterized by the proto-derivative of the solution mapping associated with the nonlinear obstacle plate problem, in terms of the solution of a variational inequality.
\end{abstract}

Mathematics Subject Classification. 49A29, 90C31, 74B20, 74K20.

Received December 27, 2000. Revised July 12, 2001.

\section{INTRODUCTION}

The shape sensitivity analysis is a subject of extremely importance in shape optimization. In continuum mechanics this analysis can be done applying the material derivative concept, as for example, in the case of the linear obstacle plate problem $[9,10]$, where the properties of differentiability of projections on polyhedric sets $[4,8]$ are used. However, this methodology can not be used to derive the sensitivity result for the nonlinear obstacle plate problem. In fact, for the linear case the solution is unique, and it can be characterized as the projection of the force acting on the plate, on the constraint set defined by the obstacle. For the nonlinear obstacle plate problem, the solution may not be unique [5] and it can not be characterized in terms of a projection on the constraint set defined by the obstacle. So the approach of $[9,10]$ is not adequate to analyse the sensitivity of the nonlinear problem. Therefore in this paper we apply another methodology which uses a generalized derivative (the proto-derivative) in order to derive the sensitivity result. The basic description of the problem, the sensitivity result and the main results are next summarized.

\footnotetext{
Keywords and phrases: Plate problem, variational inequality, sensitivity analysis.

* The support of the projects PRAXIS/PCEX/C/MAT/38/96 "Variational Models and Optimization", and Sapiens Proj99, No. 34471 "Nonlinear Partial Differential Equations and Interfaces Problems" of the Ministry of Science and Technology of Portugal are gratefully acknowledged.

${ }^{1}$ Departamento de Matemática, Universidade de Coimbra, Apartado 3008, 3000 Coimbra, Portugal;

e-mail: isabelf@mat.uc.pt

2 e-mail: carlosl@mat.uc.pt
}

(C) EDP Sciences, SMAI 2002 
Let $\left\{\Omega_{t}\right\}_{t\rangle 0}$ be a family of perturbations of a given domain $\Omega=\Omega_{0} \subset \mathbb{R}^{2}$. For each small parameter $t$ we consider a thin elastic clamped plate, with thickness $h$, independent of $t$, occupying the domain $\overline{\Omega_{t}} \times\left[-\frac{h}{2}, \frac{h}{2}\right]$, and made of a (geometrically) nonlinear Hookean material. By the action of external loads the plate may come in frictionless contact with a rigid obstacle. Let $W(t)$ be a displacement describing the state of equilibrium of the plate, whose middle plane is $\Omega_{t}$. By [5], $W(t)$ may not be unique, so $W$ can be characterized as a multifunction. The purpose is to analyse the sensitivity of $W(0)$, which is the set solution of the plate problem with middle plane $\Omega_{0}$, with respect to small perturbations $\Omega_{t}$ of the domain $\Omega_{0}$. The method presented here to obtain this sensitivity result, is based on [6], where the sensitivity of parameterized variational inequalities in Banach spaces (without uniqueness of solution) is quantified in terms of a generalized derivative, which is the (multifunction) proto-derivative $[7,11]$. As stated in Theorem 2.7, and proved in Sections 3, 4 the proto-derivative associated to the nonlinear obstacle plate problem is the multifunction $D W(0)\left(w^{0}\right):[0, \delta] \rightarrow H_{0}^{2}(\Omega)$ defined in $(2.15)$ by

$$
D W(0)\left(w^{0}\right)(t)=\left\{w \in K^{*}: \quad\left\langle-D S\left(w^{0}, 0\right)(w, t), z-w\right\rangle \leq 0, \quad \forall z \in K^{*}\right\}
$$

where $w^{0} \in W(0), D S\left(w^{0}, 0\right)$ is the semi-derivative of $S$ at $\left(w^{0}, t=0\right),(S$ defined in (1.44) is the nonlinear mapping associated to the nonlinear plate problem) and $K^{*}$ is a set defined in (2.16) by

$$
K^{*}=\overline{\left[F_{0}-S\left(w^{0}, 0\right)\right]^{\perp} \cap \cup_{\lambda\rangle 0} \lambda\left(K-w^{0}\right)} \subset H_{0}^{2}(\Omega)
$$

where the symbol ${ }^{\perp}$ means the orthogonal set, $K$ is the set related to the constraints defined by the obstacle, and $F_{0}$ is a linear operator related to the force acting on the plate. Therefore the elements of the proto-derivative are the solutions of the variational inequality defined in (0.1).

The main contribution of this paper is the proof that the two assumptions of the abstract sensitivity result of [6] are satisfied for this nonlinear plate problem. One assumption imposes that, the nonlinear mapping involved in the definition of the nonlinear plate problem, considered as a function of the middle plane $\Omega_{t}$, must be semi-differentiable at $t=0$. This property of semi-differentiability $[6,12]$ is proven in Section 4 , Propositions 4.1 and 4.2, and relies essentially on the continuity, ellipticity and differentiability properties of the nonlinear mapping, despite the nonlinearity of the problem. The other assumption obliges the constraint set defined by the obstacle to be a polyhedric set, in the sense of Definition 2.1. This definition includes an orthogonal set defined by the nonlinear mapping characterizing the problem, and it coincides with the definition of polyhedric set of $[9,10]$ for the linear obstacle plate problem. The proof of this polyhedricity assumption is done in Section 3 and is a straightforward adaptation of $[9,10]$. In fact, the definitions of polyhedric set in the linear and nonlinear cases, differ by a nonlinear term, but, this does not change substantially the arguments of $[9,10]$. By the abstract sensitivity result of $[6]$ these two conditions are enough to assure that the protoderivative of the multifunction $W(t)$ exists, at $t=0$, which originates the sensitivity result (Th. 2.7) for this particular nonlinear obstacle plate problem. Moreover, we also prove, in Section 5, that the results obtained in $[9,10]$, for the linear obstacle plate problem, may be recovered using the methodology applied to the nonlinear case, that is, Theorem 2.7 also applies to the linear case and the proto-derivative coincides with the material derivative in this case.

Finally let us briefly describe the contents of the present paper. In Section 1, the differential and variational formulations of the problem, in the perturbed domain $\Omega_{t}$, are introduced, as well as, the reformulation of the variational problem in the fixed domain $\Omega_{0}$. In Section 2 , we recall some differentiability concepts, as the semi-differentiability and the proto-differentiability, and the definition of polyhedric set. We also state, in Theorem 2.7, the sensitivity result for the nonlinear obstacle plate problem. In Sections 3 and 4 we prove that the hypotheses required in Theorem 2.7 are verified. In Section 5 we show that, for the linear obstacle plate problem the sensitivity result obtained in $[9,10]$, with a different methodology, coincides with the sensitivity result expressed in terms of the proto-derivative, using Theorem 2.7. We finally present some conclusions and future work. 


\section{Notations And DescRiption of THE PROBlem}

In this section, we firstly describe the family of perturbed domains $\left\{\Omega_{t}\right\}$, then we define the differential and variational formulations of the nonlinear obstacle plate problem, posed in $\Omega_{t}$ and finally we give the reformulation of the variational problem in the fixed domain $\Omega$.

For this purpose we must introduce some notations. Throughout the paper, the greek indices $\alpha, \beta \ldots$ belong to the set $\{1,2\}$ and the Einstein summation convention with respect to repeated index is employed, that is, $a_{\alpha} b_{\alpha}=\sum_{\alpha=1}^{2} a_{\alpha} b_{\alpha}$. We also denote by $c d$ and $c: d$ the outer and inner product, respectively, of tensors $c$ and $d$. For example, if $c_{\alpha \beta}$ and $d_{\lambda \mu}$ are the components of the second order tensors $c$ and $d$, respectively, then, $e=c d$ is a fourth order tensor with components $e_{\alpha \beta \lambda \mu}=c_{\alpha \beta} d_{\lambda \mu}$ and $c: d=\sum_{\alpha, \beta=1}^{2} c_{\alpha \beta} d_{\alpha \beta}$. Moreover, if $P$ and $Q$ are two matrices we denote by $P . Q$ the matrix multiplication of $P$ by $Q$. The transpose of matrix $P$ is denoted by $P^{T}$.

\subsection{The perturbed domain $\Omega_{t}$}

Let $\Omega$ be an open, bounded and connected subset of $\mathbb{R}^{2}$, with a Lipschtiz boundary $\partial \Omega$. We introduce a family of perturbations $\left\{\Omega_{t}\right\}$ of $\Omega$, for $t \in[0, \delta]$, with $\delta$ a small parameter, defined as follows: for each $t, \Omega_{t}$ is the range of the transformation $T_{t}$ in $\Omega$

$$
\begin{aligned}
T_{t}: & \mathbb{R}^{2} \longrightarrow \mathbb{R}^{2} \\
& x \longrightarrow T_{t}(x)=(I+t \theta)(x)=x_{t}
\end{aligned}
$$

where $I$ is the identity mapping in $\mathbb{R}^{2}$, and $\theta: \mathbb{R}^{2} \rightarrow \mathbb{R}^{2}$ is a smooth enough mapping, at least $\theta \in\left[W^{2, \infty}\left(\mathbb{R}^{2}\right)\right]^{2}$. By definiton

$$
\Omega_{t}=T_{t}(\Omega), \quad t \in[0, \delta]
$$

and in particular the fixed domain $\Omega$ is equal to $\Omega_{0}$. Moreover, with the definition of $T_{t}$, we conclude that $\Omega_{t}$ is a perturbation of $\Omega$ in the direction of the vector field $\theta$.

\subsection{Formulation of the nonlinear obstacle plate problem in $\Omega_{t}$}

The differential formulation of the nonlinear obstacle plate problem, for a plate with middle plane $\Omega_{t}$ is the following [5]:

$$
\begin{aligned}
& \text { Find } \quad\left(\underline{u}_{t}, w_{t}\right): \Omega_{t} \subset \mathbb{R}^{2} \rightarrow \mathbb{R}^{3}, \quad \text { such that: } \\
& D \Delta^{2} w_{t}-h\left[\sigma_{\alpha \beta}\left(\underline{u}_{t}, w_{t}\right) w_{t, \beta}\right]_{, \alpha} \geq f, \quad \text { in } \Omega_{t}, \\
& w_{t} \geq \psi, \quad \text { in } \Omega_{t}, \\
& \left(D \Delta^{2} w_{t}-h\left[\sigma_{\alpha \beta}\left(\underline{u}_{t}, w_{t}\right) w_{t, \beta}\right]_{, \alpha}-f\right)\left(w_{t}-\psi\right)=0, \quad \text { in } \Omega_{t}, \\
& \sigma_{\alpha \beta}\left(\underline{u}_{t}, w_{t}\right)_{, \beta}=0, \quad \text { in } \quad \Omega_{t}, \\
& \underline{u}_{t}=0, \quad w_{t}=\frac{\partial w_{t}}{\partial n}=0, \quad \text { in } \partial \Omega_{t} .
\end{aligned}
$$

In (1.3-1.8) the set $\Omega_{t}$ represents the middle plane of the undeformed thin plate. For each point $x_{t}=\left(x_{t 1}, x_{t 2}\right) \in$ $\Omega_{t}$ we denote by $\underline{u}_{t}\left(x_{t}\right)=\left(u_{t 1}\left(x_{t}\right), u_{t 2}\left(x_{t}\right)\right)$ and $w_{t}\left(x_{t}\right)$ the horizontal and vertical displacements of $x_{t}$, respectively. The plate is subjected to a transverse load of intensity $f$, per unit of area of the middle plane. The shape of the obstacle is given by the prescribed function $\psi$. The functions $f: \mathbb{R}^{2} \rightarrow \mathbb{R}$ and $\psi: \mathbb{R}^{2} \rightarrow \mathbb{R}$ are assumed smooth enough. The constant $D=\frac{E h^{3}}{12\left(1-\nu^{2}\right)}$ is the flexural rigidity of the plate with $E$ the modulus of elasticity, 
$\nu$ the Poisson ratio and $h$ the thickness of the plate. The conditions (1.8) are the boundary conditions for a clamped plate. Moreover $\Delta^{2}$ is the biharmonic operator, $\frac{\partial}{\partial n}$ is the normal operator and the index notation $\cdot, \alpha$ means partial derivative with respect to $x_{t \alpha}$. Finally, $\sigma\left(\underline{u}_{t}, w_{t}\right)=\left(\sigma_{\alpha \beta}\left(\underline{u}_{t}, w_{t}\right)\right)$ is the second order membrane stress tensor defined by

$$
\sigma\left(\underline{u}_{t}, w_{t}\right)=C:\left(e\left(\underline{u}_{t}\right)+\frac{1}{2} \nabla w_{t} \nabla w_{t}\right), \quad \sigma_{\alpha \beta}\left(\underline{u}_{t}, w_{t}\right)=C_{\alpha \beta \lambda \mu}\left(e_{\lambda \mu}\left(\underline{u}_{t}\right)+\frac{1}{2} w_{t, \lambda} w_{t, \mu}\right) .
$$

The fourth order elasticity tensor $C$ has components

$$
C_{\alpha \beta \lambda \mu}=\frac{E}{2\left(1-\nu^{2}\right)}\left[(1-\nu)\left(\delta_{\alpha \lambda} \delta_{\beta \mu}+\delta_{\alpha \mu} \delta_{\beta \lambda}\right)+2 \nu \delta_{\alpha \beta} \delta_{\lambda \mu}\right]
$$

with $\delta_{\alpha \beta}$ the standard Kronecker delta notation and $e\left(\underline{u}_{t}\right)=\left(e_{\alpha \beta}\left(\underline{u}_{t}\right)\right)$ the second order strain tensor defined by

$$
e\left(\underline{u}_{t}\right)=\frac{1}{2}\left(\nabla \underline{u}_{t}+\left(\nabla \underline{u}_{t}\right)^{T}\right), \quad e_{\alpha \beta}\left(\underline{u}_{t}\right)=\frac{1}{2}\left(u_{t \alpha, \beta}+u_{t \beta, \alpha}\right) .
$$

The tensors $\nabla w_{t}$ and $\nabla \underline{u}_{t}$ are the gradients of $w_{t}$ and $\underline{u}_{t}$, which are matrices of order $1 \times 2$ and $2 \times 2$, respectively, and defined by

$$
\nabla w_{t}=\left(w_{t, \alpha}\right), \quad \nabla \underline{u}_{t}=\left(u_{t \alpha, \beta}\right)
$$

The variational formulation of the system (1.3-1.8) corresponds to the following system of variational inequality and equation [5]:

$$
\begin{aligned}
& \text { Find }\left(\underline{u}_{t}, w_{t}\right) \in\left[H_{0}^{1}\left(\Omega_{t}\right)\right]^{2} \times K_{t}: \\
& A_{t}\left(w_{t}, z_{t}-w_{t}\right)+a_{t}\left(\underline{u}_{t} ; w_{t}, z_{t}-w_{t}\right) \geq F_{t}\left(z_{t}-w_{t}\right), \quad \forall z_{t} \in K_{t}, \\
& B_{t}\left(\underline{u}_{t}, \underline{v}_{t}\right)+b_{t}\left(w_{t}, \underline{v}_{t}\right)=0, \quad \forall \underline{v}_{t} \in\left[H_{0}^{1}\left(\Omega_{t}\right)\right]^{2},
\end{aligned}
$$

where $K_{t}$ is the constraint set defined by the obstacle

$$
K_{t}=\left\{z_{t} \in H_{0}^{2}\left(\Omega_{t}\right): z_{t} \geq \psi \quad \text { in } \quad \Omega_{t}\right\}
$$

and $H_{0}^{1}\left(\Omega_{t}\right), H_{0}^{2}\left(\Omega_{t}\right)$ are the usual Sobolev spaces defined by

$$
\begin{aligned}
& H_{0}^{1}\left(\Omega_{t}\right)=\left\{v_{t} \in H^{1}\left(\Omega_{t}\right): v_{\left.t\right|_{\partial \Omega_{t}}}=0\right\} \\
& H_{0}^{2}\left(\Omega_{t}\right)=\left\{z_{t} \in H^{2}\left(\Omega_{t}\right): z_{\left.t\right|_{\partial \Omega_{t}}}=\left.\frac{\partial z_{t}}{\partial n}\right|_{\partial \Omega_{t}}=0\right\}
\end{aligned}
$$


The expressions of the forms $A_{t}, a_{t}, F_{t}, B_{t}$ and $b_{t}$ are

$$
\begin{aligned}
& {\left[\begin{array}{l}
A_{t}: H_{0}^{2}\left(\Omega_{t}\right) \times H_{0}^{2}\left(\Omega_{t}\right) \rightarrow \mathbb{R}, \\
A_{t}\left(w_{t}, z_{t}\right)=D \int_{\Omega_{t}}\left\{\nu \Delta w_{t} \Delta z_{t}+(1-\nu) w_{t, \alpha \beta} z_{t, \alpha \beta}\right\} \mathrm{d} x_{t}= \\
\frac{h^{3}}{12} \int_{\Omega_{t}} C_{\alpha \beta \lambda \mu} w_{t, \alpha \beta} z_{t, \lambda \mu} \mathrm{d} x_{t}=\frac{h^{3}}{12} \int_{\Omega_{t}} C:\left(\nabla^{2} w_{t} \nabla^{2} z_{t}\right) \mathrm{d} x_{t},
\end{array}\right.} \\
& {\left[\begin{array}{l}
B_{t}:\left[H_{0}^{1}\left(\Omega_{t}\right)\right]^{2} \times\left[H_{0}^{1}\left(\Omega_{t}\right)\right]^{2} \rightarrow \mathbb{R}, \\
B_{t}\left(\underline{u}_{t}, \underline{v}_{t}\right)=h \int_{\Omega_{t}} C_{\alpha \beta \lambda \mu} e_{\alpha \beta}\left(\underline{u}_{t}\right) e_{\lambda \mu}\left(\underline{v}_{t}\right) \mathrm{d} x_{t}=h \int_{\Omega_{t}} C:\left(e\left(\underline{u}_{t}\right) e\left(\underline{v}_{t}\right)\right) \mathrm{d} x_{t},
\end{array}\right.} \\
& {\left[\begin{array}{l}
a_{t}:\left[H_{0}^{1}\left(\Omega_{t}\right)\right]^{2} \times H_{0}^{2}\left(\Omega_{t}\right) \times H_{0}^{2}\left(\Omega_{t}\right) \rightarrow \mathbb{R} \\
a_{t}\left(\underline{u}_{t} ; w_{t}, z_{t}\right)=h \int_{\Omega_{t}} \sigma_{\alpha \beta}\left(\underline{u}_{t}, w_{t}\right) w_{t, \alpha} z_{t, \beta} \mathrm{d} x_{t}=
\end{array}\right.} \\
& h \int_{\Omega_{t}} C:\left(\left[e\left(\underline{u}_{t}\right)+\frac{1}{2} \nabla w_{t} \nabla w_{t}\right] \nabla w_{t} \nabla z_{t}\right) \mathrm{d} x_{t}, \\
& {\left[\begin{array}{l}
b_{t}: H_{0}^{2}\left(\Omega_{t}\right) \times\left[H_{0}^{1}\left(\Omega_{t}\right)\right]^{2} \rightarrow \mathbb{R}, \\
b_{t}\left(w_{t}, \underline{v}_{t}\right)=\frac{h}{2} \int_{\Omega_{t}} C_{\alpha \beta \lambda \mu} w_{t, \lambda} w_{t, \mu} e_{\alpha \beta}\left(\underline{v}_{t}\right) \mathrm{d} x_{t}=\frac{h}{2} \int_{\Omega_{t}} C:\left(e\left(\underline{v}_{t}\right) \nabla w_{t} \nabla w_{t}\right) \mathrm{d} x_{t},
\end{array}\right.} \\
& {\left[\begin{array}{l}
F_{t}: H_{0}^{2}\left(\Omega_{t}\right) \rightarrow \mathbb{R} \\
F_{t}\left(w_{t}\right)=\int_{\Omega_{t}} f w_{t} \mathrm{~d} x_{t} .
\end{array}\right.}
\end{aligned}
$$

In (1.18), $\nabla^{2} w_{t}=\left(w_{t, \alpha \beta}\right)$ and $\nabla^{2} z_{t}=\left(z_{t, \alpha \beta}\right)$ are the matrices of the second derivatives of the scalar functions $w_{t}$ and $z_{t}$, respectively. The symbol $\Delta$ denotes the laplacian, that is, for example, $\Delta w_{t}=w_{t, \alpha \alpha}$.

It is easy to verify that the horizontal displacement $\underline{u}_{t}$ can be eliminated from the variational formulation $(1.13,1.14)$. In fact, by the Lax-Milgram theorem, Equation (1.14) has a unique solution, for each $w_{t}$. So there is a mapping

$$
\begin{aligned}
& G_{t}: H_{0}^{2}\left(\Omega_{t}\right) \longrightarrow\left[H_{0}^{1}\left(\Omega_{t}\right)\right]^{2} \\
& w_{t} \longrightarrow G_{t}\left(w_{t}\right) \text {, }
\end{aligned}
$$

such that $G_{t}\left(w_{t}\right)$ is the solution of the equation

$$
B_{t}\left(G_{t}\left(w_{t}\right), \underline{v}_{t}\right)=-b_{t}\left(w_{t}, \underline{v}_{t}\right), \quad \forall \underline{v}_{t} \in\left[H_{0}^{1}\left(\Omega_{t}\right)\right]^{2} .
$$

So $(1.13,1.14)$ is equivalent to the following nonlinear variational inequality

$$
\left\{\begin{array}{l}
\text { Find } w_{t} \in K_{t} \\
A_{t}\left(w_{t}, z_{t}-w_{t}\right)+a_{t}\left(G_{t}\left(w_{t}\right) ; w_{t}, z_{t}-w_{t}\right) \geq F_{t}\left(z_{t}-w_{t}\right), \quad \forall z_{t} \in K_{t} .
\end{array}\right.
$$

The existence of solution of (1.25) is based on an existence lemma for nonlinear operators [1] and relies on the fact that the operator involved in the definition of inequality (1.25) is coercive and the sum of a monotone operator with a completely continuous operator [5].

\subsection{Variational problem posed in the fixed domain $\Omega$}

The nonlinear obstacle plate problem (1.25) posed in $\Omega_{t}$, can be transported to the fixed domain $\Omega$, using the transformation $T_{t}$. In order to do this we first express the forms $A_{t}, B_{t}, a_{t}, b_{t}$ and $F_{t}$ defined in (1.18-1.22) 
in the domain $\Omega$. We observe that

$$
\begin{aligned}
& \nabla T_{t}=I+t \nabla \theta, \quad \nabla T_{t}^{-1}=I-t \nabla \theta+\mathcal{O}\left(t^{2}\right) \\
& \nabla^{2} T_{t}^{-1}=-t \nabla^{2} \theta+\mathcal{O}\left(t^{2}\right) \\
& \operatorname{det} \nabla T_{t}=1+t \operatorname{div} \theta+t^{2} \operatorname{det} \nabla \theta
\end{aligned}
$$

The second order matrices $\nabla T_{t}, \nabla T_{t}^{-1}$ and $\nabla \theta$ are the gradients of $T_{t}, T_{t}^{-1}$ and $\theta$, respectively, $I$ is the identity matrix of order $2, \mathcal{O}\left(t^{2}\right)$ means a term that verifies $\left\|\mathcal{O}\left(t^{2}\right)\right\|_{\left[W^{2, \infty}\left(\mathbb{R}^{2}\right)\right]^{2}} \leq c t^{2}$, with $c$ a constant independent of $t, \nabla^{2} T_{t}^{-1}$ and $\nabla^{2} \theta$ are the second order matrices of the second derivatives of $T_{t}^{-1}$ and $\theta$, respectively. For example, if $\theta=\left(\theta_{1}, \theta_{2}\right)$ we have $\nabla^{2} \theta=\left(\nabla^{2} \theta_{1} \nabla^{2} \theta_{2}\right)^{T}$. The divergence of $\theta$ is denoted by $\operatorname{div} \theta=\theta_{\alpha, \alpha}$. Finally, $\operatorname{det} \nabla T_{t}$ and $\operatorname{det} \nabla \theta$ are the determinants of matrices $\nabla T_{t}$ and $\nabla \theta$, respectively.

To each function $z_{t}$ or $\underline{v}_{t}=\left(v_{t 1}, v_{t 2}\right)$ defined in $\Omega_{t}$ we associate the corresponding functions $z^{t}$ or $\underline{v}^{t}=\left(v_{1}^{t}, v_{2}^{t}\right)$ defined in $\Omega$ by

$$
z^{t}=z_{t} \circ T_{t}, \quad \underline{v}^{t}=\underline{v}_{t} \circ T_{t} .
$$

Then we immediately obtain from (1.26),

$$
\nabla z_{t}=\nabla z^{t} \cdot \nabla T_{t}^{-1}=\nabla z^{t}-t \nabla z^{t} \cdot \nabla \theta+\mathcal{O}\left(t^{2}\right),
$$

where the point . means matrix multiplication and $\mathcal{O}\left(t^{2}\right)$ is a term that verifies $\left\|\mathcal{O}\left(t^{2}\right)\right\|_{H_{0}^{2}(\Omega)} \leq c t^{2}\left\|z^{t}\right\|_{H_{0}^{2}(\Omega)}$, with $c$ a constant independent of $t$. Also because $e\left(\underline{v}_{t}\right)=\frac{1}{2}\left(\nabla \underline{v}_{t}+\nabla \underline{v}_{t}^{T}\right)$, then

$$
e\left(\underline{v}_{t}\right)=e\left(\underline{v}^{t}\right)-t \hat{e}\left(\underline{v}^{t}, \theta\right)+\mathcal{O}\left(t^{2}\right), \quad \text { with } \quad \hat{e}\left(\underline{v}^{t}, \theta\right)=\frac{1}{2}\left(\nabla \underline{v}^{t} \cdot \nabla \theta+(\nabla \theta)^{T} \cdot\left(\nabla \underline{v}^{t}\right)^{T}\right),
$$

where $\hat{e}\left(\underline{v}^{t}, \theta\right)$ depends linearly on $\underline{v}^{t}$ and $\mathcal{O}\left(t^{2}\right)$ is a term that verifies $\left\|\mathcal{O}\left(t^{2}\right)\right\|_{\left[H_{0}^{1}(\Omega)\right]^{2}} \leq c t^{2}\left\|\underline{v}^{t}\right\|_{\left[H_{0}^{1}(\Omega)\right]^{2}}$, with $c$ a constant independent of $t$.

By the chain rule derivative we can relate $\nabla^{2} z_{t}=\left(z_{t, \alpha \beta}\right)$ with $\nabla^{2} z^{t}=\left(z_{, \alpha \beta}^{t}\right)$, by the following matrix equation

$$
\nabla^{2} z_{t}=\left(\nabla T_{t}^{-1}\right)^{T} \cdot \nabla^{2} z^{t} \cdot \nabla T_{t}^{-1}+\left[\begin{array}{cc}
\nabla z^{t} & 0 \\
0 & \nabla z^{t}
\end{array}\right] \cdot\left[\begin{array}{c}
\nabla^{2} T_{t}^{-1} \\
\nabla^{2} T_{t}^{-1}
\end{array}\right]
$$

where 0 means the zero matrix of order $1 \times 2$. So using $(1.26)$ in $(1.30)$ we deduce that

$$
\left\{\begin{array}{l}
\nabla^{2} z_{t}=\nabla^{2} z^{t}-t \hat{d}\left(\theta, z^{t}\right)+\mathcal{O}\left(t^{2}\right) \\
\hat{d}\left(\theta, z^{t}\right)=(\nabla \theta)^{T} \cdot \nabla^{2} z^{t}+\nabla^{2} z^{t} \cdot \nabla \theta+\left[\begin{array}{c}
\nabla z^{t} \cdot \nabla^{2} \theta \\
\nabla z^{t} \cdot \nabla^{2} \theta
\end{array}\right],
\end{array}\right.
$$

where $\hat{d}\left(\theta, z^{t}\right)$ is a matrix of order 2 that depends linearly on $z^{t}$ and $\mathcal{O}\left(t^{2}\right)$ is a term that verifies $\left\|\mathcal{O}\left(t^{2}\right)\right\|_{H_{0}^{2}(\Omega)} \leq$ $c t^{2}\left\|z^{t}\right\|_{H_{0}^{2}(\Omega)}$, with $c$ a constant independent of $t$.

Now, with the formula of $\operatorname{det} \nabla T_{t}$ and formulas $(1.28,1.29)$ and $(1.31)$ we can write the integrals of $(1.18-1.22)$ in $\Omega$. We have

$$
\left\{\begin{array}{l}
A_{t}\left(w_{t}, z_{t}\right)=A_{0}\left(w^{t}, z^{t}\right)-t A_{1}\left(w^{t}, z^{t}\right)+\mathcal{O}_{A}\left(t^{2}\right) \\
B_{t}\left(\underline{u}_{t}, \underline{v}_{t}\right)=B_{0}\left(\underline{u}^{t}, \underline{v}^{t}\right)-t B_{1}\left(\underline{u}^{t}, \underline{v}^{t}\right)+\mathcal{O}_{B}\left(t^{2}\right) \\
a_{t}\left(\underline{u}_{t} ; w_{t}, z_{t}\right)=a_{0}\left(\underline{u}^{t} ; w^{t}, z^{t}\right)-t a_{1}\left(\underline{u}^{t} ; w^{t}, z^{t}\right)+\mathcal{O}_{a}\left(t^{2}\right) \\
b_{t}\left(w_{t}, \underline{v}_{t}\right)=b_{0}\left(w^{t}, \underline{v}^{t}\right)-t b_{1}\left(w^{t}, \underline{v}^{t}\right)+\mathcal{O}_{b}\left(t^{2}\right) \\
F_{t}\left(w_{t}\right)=F_{0}\left(w^{t}\right)+t F_{1}\left(w^{t}\right)+\mathcal{O}_{F}\left(t^{2}\right) .
\end{array}\right.
$$


where the scalars terms $\mathcal{O}_{A}\left(t^{2}\right), \mathcal{O}_{B}\left(t^{2}\right), \mathcal{O}_{a}\left(t^{2}\right), \mathcal{O}_{b}\left(t^{2}\right)$ and $\mathcal{O}_{F}\left(t^{2}\right)$ verify

$$
\begin{aligned}
& \left|\mathcal{O}_{A}\left(t^{2}\right)\right| \leq c t^{2}\left\|w^{t}\right\|_{H_{0}^{2}(\Omega)}|| z^{t} \|_{H_{0}^{2}(\Omega)} \\
& \left|\mathcal{O}_{B}\left(t^{2}\right)\right| \leq c t^{2}\left\|\underline{u}^{t}\right\|_{\left[H_{0}^{1}(\Omega)\right]^{2}}\left\|\underline{v}^{t}\right\|_{\left[H_{0}^{1}(\Omega)\right]^{2}} \\
& \left|\mathcal{O}_{a}\left(t^{2}\right)\right| \leq c t^{2}\left\|\underline{u}^{t}\right\|_{\left[H_{0}^{1}(\Omega)\right]^{2}}\left\|w^{t}\right\|_{H_{0}^{2}(\Omega)}\left\|z^{t}\right\|_{H_{0}^{2}(\Omega)} \\
& \left|\mathcal{O}_{b}\left(t^{2}\right)\right| \leq c t^{2}\left\|w^{t}\right\|_{H_{0}^{2}(\Omega)}\left\|\underline{v}^{t} \mid\right\|_{\left[H_{0}^{1}(\Omega)\right]^{2}} \\
& \left|\mathcal{O}_{F}\left(t^{2}\right)\right| \leq c t^{2}\left\|w^{t}\right\|_{L^{2}(\Omega)}
\end{aligned}
$$

with $c$ different constants independent of $t$, but depending on $\theta$.

For the decomposition of $A_{t}$

$$
\left\{\begin{array}{l}
A_{0}\left(w^{t}, z^{t}\right)=\frac{h^{3}}{12} \int_{\Omega} C:\left(\nabla^{2} w^{t} \nabla^{2} z^{t}\right) \mathrm{d} x \\
A_{1}\left(w^{t}, z^{t}\right)=\frac{h^{3}}{12} \int_{\Omega} C:\left(-\nabla^{2} w^{t} \nabla^{2} z^{t} \operatorname{div} \theta+\nabla^{2} w^{t} \hat{d}\left(\theta, z^{t}\right)+\hat{d}\left(\theta, w^{t}\right) \nabla^{2} z^{t}\right) \mathrm{d} x,
\end{array}\right.
$$

and $A_{1}$ is a bilinear form. For the decomposition of $B_{t}$

$$
\left\{\begin{array}{l}
B_{0}\left(\underline{u}^{t}, \underline{v}^{t}\right)=h \int_{\Omega} C:\left(e\left(\underline{u}^{t}\right) e\left(\underline{v}^{t}\right)\right) \mathrm{d} x \\
B_{1}\left(\underline{u}^{t}, \underline{v}^{t}\right)=h \int_{\Omega} C:\left(e\left(\underline{u}^{t}\right) \hat{e}\left(\underline{v}^{t}, \theta\right)+\hat{e}\left(\underline{u}^{t}, \theta\right) e\left(\underline{v}^{t}\right)-e\left(\underline{u}^{t}\right) e\left(\underline{v}^{t}\right) \operatorname{div} \theta\right) \mathrm{d} x,
\end{array}\right.
$$

with $B_{1}$ a bilinear form. For the decomposition of $a_{t}$

$$
\left\{\begin{array}{l}
a_{0}\left(\underline{u}^{t} ; w^{t}, z^{t}\right)=h \int_{\Omega} C:\left\{e\left(\underline{u}^{t}\right)+\frac{1}{2} \nabla w^{t} \nabla w^{t}\right\} \nabla w^{t} \nabla z^{t} \mathrm{~d} x \\
a_{1}\left(\underline{u}^{t} ; w^{t}, z^{t}\right)=h \int_{\Omega} C:\left\{e\left(\underline{u}^{t}\right) \nabla w^{t}\left(-\nabla z^{t} \operatorname{div} \theta+\nabla z^{t} \nabla \theta+\nabla \theta \nabla z^{t}\right)\right. \\
+\hat{e}\left(\underline{u}^{t}, \theta\right) \nabla w^{t} \nabla z^{t}+\frac{1}{2}\left[\nabla w^{t} \nabla w^{t} \nabla w^{t}\left(\nabla z^{t} \nabla \theta+\nabla \theta \nabla z^{t}+\nabla z^{t} \operatorname{div} \theta\right)+\right. \\
\left.\left.\left(\nabla w^{t} \nabla w^{t} \nabla \theta+\nabla w^{t} \nabla \theta \nabla w^{t}\right) \nabla w^{t} \nabla z^{t}\right]\right\} \mathrm{d} x .
\end{array}\right.
$$

For the decomposition of $b_{t}$

$$
\left\{\begin{array}{l}
b_{0}\left(w^{t}, \underline{v}^{t}\right)=\frac{h}{2} \int_{\Omega} C:\left(e\left(\underline{v}^{t}\right) \nabla w^{t} \nabla w^{t}\right) \mathrm{d} x \\
b_{1}\left(w^{t}, \underline{v}^{t}\right)=\frac{h}{2} \int_{\Omega} C:\left\{-e\left(\underline{v}^{t}\right) \nabla w^{t} \nabla w^{t} \operatorname{div} \theta+e\left(\underline{v}^{t}\right) \nabla w^{t} \nabla \theta \nabla w^{t}+\right. \\
\left.\hat{e}\left(\underline{v}^{t}, \theta\right) \nabla w^{t} \nabla w^{t}+e\left(\underline{v}^{t}\right) \nabla w^{t} \nabla w^{t} \nabla \theta\right\} \mathrm{d} x .
\end{array}\right.
$$

Finally for the decomposition of $F_{t}$

$$
\left\{\begin{array}{l}
F_{0}\left(w_{t}\right)=\int_{\Omega} f w^{t} \mathrm{~d} x \\
F_{1}\left(w_{t}\right)=\int_{\Omega}\left((\nabla f)^{T} \cdot \theta+f \operatorname{div} \theta\right) w^{t} \mathrm{~d} x
\end{array}\right.
$$


because, as $f$ is smooth enough, for example $f \in C^{2}\left(\mathbb{R}^{2}\right)$, we can apply the Taylor-Young formula to $f \circ T_{t}$, which gives for any $x \in \Omega$

$$
f \circ T_{t}(x)=f(x+t \theta(x))=f(x)+t(\nabla f(x))^{T} \cdot \theta(x)+\mathcal{O}\left(t^{2}\right)
$$

where the term $\mathcal{O}\left(t^{2}\right)$ satisfies $\left|\mathcal{O}\left(t^{2}\right)\right| \leq c t^{2}$, with $c$ a constant independent of $t$. With the definition (1.27) the set of constraints $K_{t}$ in (1.15) becomes $K^{t}$ defined by

$$
K^{t}=\left\{z \in H_{0}^{2}(\Omega): z \geq \psi^{t}=\psi \circ T_{t} \quad \text { in } \quad \Omega\right\} .
$$

Therefore, the nonlinear obstacle problem (1.25) is equivalent to the following variational inequality posed in $\Omega$ :

$$
\left\{\begin{array}{l}
\text { Find } w^{t} \in K^{t}: \\
A_{0}\left(w^{t}, z^{t}-w^{t}\right)-t A_{1}\left(w^{t}, z^{t}-w^{t}\right)+ \\
a_{0}\left(G_{t}\left(w_{t}\right)^{t} ; w^{t}, z^{t}-w^{t}\right)-t a_{1}\left(G_{t}\left(w_{t}\right)^{t} ; w^{t}, z^{t}-w^{t}\right) \\
\geq F_{0}\left(z^{t}-w^{t}\right)+t F_{1}\left(z^{t}-w^{t}\right)+\mathcal{O}\left(t^{2}\right), \quad \forall z^{t} \in K^{t},
\end{array}\right.
$$

where $\mathcal{O}\left(t^{2}\right)=-\mathcal{O}_{A}\left(t^{2}\right)-\mathcal{O}_{a}\left(t^{2}\right)+\mathcal{O}_{F}\left(t^{2}\right)$ and with

$$
w_{t}=w^{t} \circ T_{t}^{-1}, \quad G_{t}\left(w_{t}\right)^{t}=G_{t}\left(w_{t}\right) \circ T_{t}
$$

and $G_{t}\left(w_{t}\right)^{t}$ is the solution of

$$
\left\{\begin{array}{l}
B_{0}\left(G_{t}\left(w_{t}\right)^{t}, \underline{v}\right)-t B_{1}\left(G_{t}\left(w_{t}\right)^{t}, \underline{v}\right)= \\
-b_{0}\left(w^{t}, \underline{v}\right)+t b_{1}\left(w^{t}, \underline{v}\right)+\mathcal{O}\left(t^{2}\right), \quad \forall \underline{v} \in\left[H_{0}^{1}(\Omega)\right]^{2}
\end{array}\right.
$$

(with $\mathcal{O}\left(t^{2}\right)=-\mathcal{O}_{B}\left(t^{2}\right)-\mathcal{O}_{b}\left(t^{2}\right)$ ) which corresponds to equation (1.24), using the decompositions (1.35) and (1.37) of $B_{t}$ and $b_{t}$, respectively. Defining the mapping $S: H_{0}^{2}(\Omega) \times[0, \delta] \rightarrow\left[H_{0}^{2}(\Omega)\right]^{\prime}$, with range in the dual space of $H_{0}^{2}(\Omega)$, by

$$
\left\{\begin{array}{l}
\langle S(w, t), z\rangle=A_{0}(w, z)-t A_{1}(w, z)+a_{0}\left(G_{t}\left(w_{t}\right)^{t} ; w, z\right)- \\
t a_{1}\left(G_{t}\left(w_{t}\right)^{t} ; w, z\right)-t F_{1}(z)+\mathcal{O}\left(t^{2}\right), \quad \forall(w, t) \in H_{0}^{2}(\Omega) \times[0, \delta], \quad \forall z \in H_{0}^{2}(\Omega),
\end{array}\right.
$$

with $w_{t}=w \circ T_{t}^{-1}$ and $\mathcal{O}\left(t^{2}\right)$ the symmetric of the term $\mathcal{O}\left(t^{2}\right)$ defined in (1.41), we immediately obtain that (1.41) is equivalent to

$$
\left\{\begin{array}{l}
\text { Find } w^{t} \in K^{t}: \\
\left\langle F_{0}-S\left(w^{t}, t\right), z^{t}-w^{t}\right\rangle \leq 0, \quad \forall z^{t} \in K^{t},
\end{array}\right.
$$

where $F_{0}$ is defined in (1.38).

We finish this section with a proposition that states that the solution of (1.45) can be obtained by solving an analogous problem posed in the set

$$
K=\left\{z \in H_{0}^{2}(\Omega): z \geq 0 \quad \text { in } \quad \Omega\right\},
$$

which is independent of $t$. The objective of this proposition is to show that the variational inequality (1.45), defining the nonlinear obstacle plate problem in $\Omega$, with a constraint set dependent of $t$, can be reduced to a variational inequality whose constraint set is independent of the parameter $t$. This is crucial in order to apply the abstract result of [6] for parameterized variational inequalities, where the constraint set is independent of the parameters. 
Proposition 1.1. The function $w^{t} \in K^{t}$ is a solution of problem (1.45) if and only if

$$
w^{t}=\varphi^{t}+\psi^{t}
$$

where $\varphi^{t} \in K$ is the solution of the following problem

$$
\left\{\begin{array}{l}
\text { Find } \varphi^{t} \in K: \\
\left\langle F_{0}-V\left(\varphi^{t}, t\right), z-\varphi^{t}\right\rangle \leq 0, \quad \forall z \in K,
\end{array}\right.
$$

where

$$
\left\{\begin{array}{l}
\langle V(\varphi, t), z\rangle=\left\langle S\left(\varphi+\psi^{t}, t\right), z\right\rangle= \\
A_{0}\left(\varphi+\psi^{t}, z\right)-t A_{1}\left(\varphi+\psi^{t}, z\right)+a_{0}\left(G_{t}\left(\varphi_{t}+\psi\right)^{t} ; \varphi+\psi^{t}, z\right) \\
-t a_{1}\left(G_{t}\left(\varphi_{t}+\psi\right)^{t} ; \varphi+\psi^{t}, z\right)-t F_{1}(z)+\mathcal{O}\left(t^{2}\right), \\
\forall(\varphi, t) \in H_{0}^{2}(\Omega) \times[0, \delta], \quad \forall z \in H_{0}^{2}(\Omega) \quad \text { and } \quad \varphi_{t}=\varphi \circ T_{t}^{-1} .
\end{array}\right.
$$

Proof. Suppose that $\varphi^{t}$ is a solution of (1.45), then $w^{t}=\varphi^{t}+\psi^{t} \in K^{t}$. Using the definitions of $S$ and $V$, and because the mapping $z \rightarrow z+\psi^{t}$ is a bijection between the sets $K$ and $K^{t}$ we have the following equivalences:

$$
\begin{aligned}
& \left\langle F_{0}-V\left(\varphi^{t}, t\right), z-\varphi^{t}\right\rangle \leq 0, \quad \forall z \in K \Longleftrightarrow \\
& \left\langle F_{0}-S\left(\varphi^{t}+\psi^{t}, t\right), z+\psi^{t}-\left(\varphi^{t}+\psi^{t}\right)\right\rangle \leq 0, \quad \forall z \in K \Longleftrightarrow \\
& \left.\left\langle F_{0}-S\left(w^{t}, t\right), z^{t}-w^{t}\right)\right\rangle \leq 0, \quad \forall z^{t} \in K^{t},
\end{aligned}
$$

which proves the result.

Remark 1.2. By this proposition we conclude that it is enough to solve (1.48) in order to determine the solution of (1.45). In the following sections we always consider problem (1.48) and the special case $\psi^{t}=0$, that is $\psi=0$, which means that the problem that will be considered is

$$
\left\{\begin{array}{l}
\text { Find } w^{t} \in K: \\
\left\langle F_{0}-S\left(w^{t}, t\right), z-w^{t}\right\rangle \leq 0, \quad \forall z \in K
\end{array}\right.
$$

where $S$ and $K$ are defined by (1.44) and (1.46), respectively. The reason of the choice $\psi=0$ is just to simplify the calculus of a semi-derivative, that is done in Section 4. In fact, as is observed in Remarks 3.5 and 4.3, the results of Sections 3,4 still hold for the more general problem $(1.48,1.49)$ with $\psi^{t} \neq 0$. This makes possible to compute the sensitivity of $w^{t}$, solution of (1.45).

\section{Differentiability and POlyhedricity CONCEPts. Main Result}

As mentioned at the end of the last section, the problem we want to analyse is the sensitivity of the solutions $w^{t} \in K$ of the variational inequality (1.51) with respect to $t$, near $t=0$. In (1.51) $F_{0}$ is given in the dual space $\left[H_{0}^{2}(\Omega)\right]^{\prime}, t$ is a parameter in the Banach space $[0, \delta], S: H_{0}^{2}(\Omega) \times[0, \delta] \rightarrow\left[H_{0}^{2}(\Omega)\right]^{\prime}$ is a single-valued mapping defined by (1.44), and finally $K \subset H_{0}^{2}(\Omega)$ is a closed, nonempty, convex set defined in (1.46). As the solution $w^{t}$ of (1.51) may not be unique, the study of the sensitivity of $w^{t}$, corresponds to the sensitivity of the multifunction $W:[0, \delta] \rightarrow H_{0}^{2}(\Omega)$ defined by

$$
\left.W(t)=\left\{w^{t} \in K: \quad\left\langle F_{0}-S\left(w^{t}, t\right), z-w^{t}\right)\right\rangle \leq 0, \quad \forall z \in K\right\} .
$$

The quantification of the sensitivity of $W$ with respect to $t$ in the neighbourhood of $t=0$, may be analysed in terms of a generalized derivative, which is the multifunction proto-derivative, as is proved in [6]. In order to describe this sensitivity result for the multifunction $W(t)$, in Theorem 2.7 , we need to introduce the concepts 
of polyhedric set, semi-derivative and proto-derivative. The concept of polyhedric set [6] in infinite-dimension is a generalization of finite-dimensional polyhedral set and its definition is the following:

Definition 2.1. A subset $K$ of a Banach space is called polyhedric at $x \in K$, for $x^{*} \in X^{\prime}$ (dual of $X$ ) if the identity holds

$$
\overline{\left(x^{*}\right)^{\perp} \cap \cup_{\lambda\rangle_{0}} \lambda(K-x)}=\left(x^{*}\right)^{\perp} \cap \overline{\cup_{\lambda\rangle 0} \lambda(K-x)},
$$

where

$$
\left(x^{*}\right)^{\perp}=\left\{y \in X: \quad\left\langle x^{*}, y\right\rangle=0\right\}
$$

and the overbar denotes the strong closure of the set.

The concept of semi-derivative $[6,12]$, which is related with the notion of directional derivative, is defined below:

Definition 2.2. A continuous function $S: X \rightarrow Y$ between two Banach spaces $X$ and $Y$ is semi-differentiable at $\bar{x}$, with semi-derivative $D S(\bar{x}): X \rightarrow Y$, if for every $\phi: \mathbb{R}^{+} \rightarrow X$, for which $\frac{\phi(s)-\bar{x}}{s}$ converges strongly to some point $x \in X$, as $s \rightarrow 0^{+}$, then

$$
D S(\bar{x})(x)=\lim _{s \rightarrow 0^{+}} \frac{S(\phi(s))-S(\bar{x})}{s} \text { in } Y \text { strongly. }
$$

For the definition of proto-derivative of a multifunction, we need the definition of graph convergence [7]:

Definition 2.3. A family of multifunctions $\left\{V_{s}\right\}$ parameterized by $\left.s\right\rangle 0$ and mapping $X$ into $Y$, with $X$ and $Y$ Banach spaces, graph converges to the multifunction $V: X \rightarrow Y$, when $s \rightarrow 0^{+}$if

$$
\lim _{s \rightarrow 0^{+}} \sup \operatorname{gph} V_{s}=\lim _{s \rightarrow 0^{+}} \inf \operatorname{gph} V_{s}=\operatorname{gph} V,
$$

where $\operatorname{gph} V_{s}(\operatorname{or} \operatorname{gph} V)$ denotes the graph of $V_{s}$ (or the graph of $V$ ), that is

$$
\operatorname{gph} V_{s}=\left\{\left(x, V_{s}(x)\right): \quad x \in X\right\},
$$

and $\lim _{s \rightarrow 0^{+}} \operatorname{infgph} V_{s}$ is the set

$$
\left\{(x, y): \forall\left(s_{n}\right), s_{n} \rightarrow 0^{+}, \quad(x, y)=\lim _{n \rightarrow \infty}\left(x_{n}, V_{s_{n}}\left(x_{n}\right)\right) \text { in } X \times Y \text { strongly, } x_{n} \in X\right\},
$$

while $\lim _{s \rightarrow 0^{+}} \operatorname{supgph} V_{s}$ is the set

$$
\left\{(x, y): \exists\left(s_{n}\right), s_{n} \rightarrow 0^{+}, \quad(x, y)=\lim _{n \rightarrow \infty}\left(x_{n}, V_{s_{n}}\left(x_{n}\right)\right) \quad \text { in } X \times Y \text { strongly, } x_{n} \in X\right\} .
$$

The following definition of proto-derivative of a multifunction, was first introduced by Rockafeller [11]:

Definition 2.4. Let $W: X \rightarrow Y$ be a multifunction and $x \in X, y \in W(x)$. If, for each $s$, the difference quotient multifunctions $\left(\Delta_{s} W\right)_{x, y}$, defined by

$$
\left(\Delta_{s} W\right)_{x, y}(\xi)=\frac{W(x+s \xi)-y}{s}, \quad \xi \in X,
$$

graph converge as $s \rightarrow 0^{+}$, then $W$ is proto-differentiable at $x$, for $y$. The proto-derivative denoted by $D W(x)(y)$ is the multifunction whose graph is the limit of the multifunctions $\left(\Delta_{s} W\right)_{x, y}$, as $s \rightarrow 0^{+}$. 
The relationship between the proto-derivative and the semi-derivative in the case of continuous functions between two Banach spaces is indicated in the following proposition [6]:

Proposition 2.5. Let $X$ and $Y$ be two Banach spaces and $S: X \rightarrow Y$.

(i) If $S$ is a continuous function and semi-differentiable at $\bar{x}$, then $S$ is proto-differentiable at $\bar{x}$, for $\bar{y}=S(\bar{x})$, with proto-derivative $D S(\bar{x})(\bar{y})$ equal to the semi-derivative $D S(\bar{x})$.

(ii) If $S$ is a multifunction and proto-differentiable at $\bar{x}$, for $\bar{y}=S(\bar{x})$, then for every $\phi: \mathbb{R}^{+} \rightarrow X$, such that

$$
\lim _{s \rightarrow 0^{+}} \frac{\phi(s)-\bar{x}}{s}=x \quad \text { in } X \text { strongly and } \lim _{s \rightarrow 0^{+}} \frac{S(\phi(s))-S(\bar{x})}{s}=y \quad \text { in } Y \text { strongly, }
$$

then the limit $y$ is in the image set of the proto-derivative $D S(\bar{x})(\bar{y})(x)$.

Using these concepts, the sensitivity result of [6] (for parameterized variational inequalities, in Banach spaces, whose solution may not be unique) is the following:

Theorem 2.6. Let $F: X \times U \rightarrow X^{\prime}$ be single-valued mapping, with $X$ and $U$ Banach spaces and consider the variational inequality

$$
\left\{\begin{array}{l}
\text { Find } \quad \bar{x} \in C: \\
\langle\bar{v}-F(\bar{x}, \bar{u}), c-\bar{x}\rangle \leq 0, \quad \forall c \in C
\end{array}\right.
$$

where $\bar{v}$ and $\bar{u}$ are fixed parameters in $X^{\prime}$ and $U$, respectively, and $\bar{x}$ is a solution of (2.11), for the fixed parameters $(\bar{v}, \bar{u})$. If $C$ is a convex set that is polyhedric at $\bar{x}$, for $\bar{v}-F(\bar{x}, \bar{u})$ and $F$ is semi-differentiable at $(\bar{x}, \bar{u})$, with semi-derivative mapping $D F(\bar{x}, \bar{u}): X \times U \rightarrow X^{\prime}$, then the solution multifunction mapping

$$
W(u, v)=\{x \in C:\langle v-F(x, u), c-x\rangle \leq 0, \quad \forall c \in C\}
$$

is proto-differentiable at $(\bar{u}, \bar{v})$ for $\bar{x}$, and $D W(\bar{u}, \bar{v})(\bar{x}): X \times U \rightarrow X^{\prime}$ is the proto-derivative mapping given by

$$
D W(\bar{u}, \bar{v})(\bar{x})(u, v)=\left\{x \in C^{*}:\langle v-D F(\bar{x}, \bar{u})(x, u), c-x\rangle \leq 0, \quad \forall c \in C^{*}\right\}
$$

where $C^{*}$ is defined by

$$
C^{*}=\overline{[\bar{v}-F(\bar{x}, \bar{u})]^{\perp} \cap \cup_{\lambda\rangle 0} \lambda(C-\bar{x})} .
$$

We can now state this theorem for the particular nonlinear obstacle plate problem considered in this paper. We remark that, as the force $F_{0}$ is fixed, the variational inequality (1.51) has only one parameter, which is $t$, and not two as in is the case of the abstract variational inequality (2.11). So, for the plate problem (1.51) Theorem 2.6 becomes:

Theorem 2.7. Let $w^{0} \in W(0)$ be a solution of problem (1.51), for $t=0$. If $K$ is a convex set, that is polyhedric at $w^{0}$, for $F_{0}-S\left(w^{0}, 0\right)$, and $S$ is semi-differentiable at $\left(w^{0}, 0\right)$, with semi-derivative mapping $D S\left(w^{0}, 0\right)$, then the multifunction mapping $W(t)$ is proto-differentiable at $t=0$, for $w^{0}$. The proto-derivative (multifunction) mapping $D W(0)\left(w^{0}\right):[0, \delta] \rightarrow H_{0}^{2}(\Omega)$ is defined by

$$
D W(0)\left(w^{0}\right)(t)=\left\{w \in K^{*}: \quad\left\langle-D S\left(w^{0}, 0\right)(w, t), z-w\right\rangle \leq 0, \quad \forall z \in K^{*}\right\},
$$

where $K^{*} \subset H_{0}^{2}(\Omega)$ and

$$
K^{*}=\overline{\left[F_{0}-S\left(w^{0}, 0\right)\right]^{\perp} \cap \cup_{\lambda>0} \lambda\left(K-w^{0}\right)} .
$$


The next two sections show that the assumptions of Theorem 2.7 are satisfied. In Section 3 we prove that the set $K$ is polyhedric, under an additional condition imposed on $F_{0}$ and consequently on $w^{0}$, and we present another characterization of $K^{*}$. In Section 4 we compute the semi-derivative $D S\left(w^{0}, 0\right)$.

\section{The COnstraint Set $K$}

The first assumption of Theorem 2.7 requires that $K$ must be a polyhedric set at $w^{0}$ for $F_{0}-S\left(w^{0}, 0\right)$, that is

$$
\overline{\left[F_{0}-S\left(w^{0}, 0\right)\right]^{\perp} \cap C_{K}\left(w^{0}\right)}=\left[F_{0}-S\left(w^{0}, 0\right)\right]^{\perp} \cap \overline{C_{K}\left(w^{0}\right)}
$$

where

$$
\left.C_{K}\left(w^{0}\right)=\cup_{\lambda>0} \lambda\left(K-w^{0}\right)=\left\{\varphi \in H_{0}^{2}(\Omega): \exists \lambda\right\rangle 0, w^{0}+\lambda \varphi \in K\right\} .
$$

We will show in Proposition 3.4, that (3.1) is satisfied under an additional condition on $F_{0}$ and $w^{0}$. The proof of this proposition is very similar to the proof of $[9,10]$, for the linear obstacle plate problem. To explain this similarity, we first remark that if

$$
S\left(w^{0}, 0\right)=A_{0}\left(w^{0}, .\right)
$$

then (3.1) becomes

$$
\overline{\left[F_{0}-A_{0}\left(w^{0}, .\right)\right]^{\perp} \cap C_{K}\left(w^{0}\right)}=\left[F_{0}-A_{0}\left(w^{0}, .\right)\right]^{\perp} \cap \overline{C_{K}\left(w^{0}\right)}
$$

where $w^{0}$ is the unique solution of the linear obstacle plate problem defined below

$$
\left\{\begin{array}{l}
\text { Find } w^{0} \in K: \\
A_{0}\left(w^{0}, z-w^{0}\right) \geq F_{0}\left(z-w^{0}\right), \quad \forall z \in K
\end{array}\right.
$$

The problem (3.5) is obtained from the nonlinear one (1.51), at $t=0$, by neglecting the nonlinear terms, in the definition of $S$ (see (5.1-5.3)). Denoting by $\Lambda: H_{0}^{2}(\Omega) \rightarrow\left[H_{0}^{2}(\Omega)\right]^{\prime}$ the isometry between $H_{0}^{2}(\Omega)$ and its dual, by the Riesz theorem, that is $\langle\Lambda w, z\rangle=A_{0}(w, z)$, for any $w$ and $z$ in $H_{0}^{2}(\Omega)$, then

$$
w^{0}=P_{K}\left(\Lambda^{-1} F_{0}\right)
$$

where the operator $P_{K}$ is the $A_{0}$-projection on the set $K$ (see (5.5)). So (3.4) is equivalent to the following set equation

$$
\overline{\left[F_{0}-P_{K}\left(\Lambda^{-1} F_{0}\right)\right]^{\perp} \cap C_{K}\left(w^{0}\right)}=\left[F_{0}-P_{K}\left(\Lambda^{-1} F_{0}\right)\right]^{\perp} \cap \overline{C_{K}\left(w^{0}\right)} .
$$

The differences between (3.1) and (3.7) are the following: in (3.1) $w^{0}$ is not the $A_{0}$-projection on the set $K$ of $\Lambda^{-1} F_{0}, w^{0}$ may not be unique and the definition of $S\left(w^{0}, 0\right)$ contains the additional nonlinear term $a_{0}\left(G_{0}\left(w^{0}\right) ; w^{0},.\right)$, that is

$$
S\left(w^{0}, 0\right)=A_{0}\left(w^{0}, .\right)+a_{0}\left(G_{0}\left(w^{0}\right) ; w^{0}, .\right) .
$$

So, (3.1) differs from (3.7) in the definition of the orthogonal set, since $C_{K}\left(w^{0}\right)$ is defined by (3.2), for both cases. For (3.1), that is, for the nonlinear case

$$
\left[F_{0}-S\left(w^{0}, 0\right)\right]^{\perp}=\left\{z \in H_{0}^{2}(\Omega):\left\langle F_{0}, z\right\rangle-A_{0}\left(w^{0}, z\right)-a_{0}\left(G_{0}\left(w^{0}\right) ; w^{0}, z\right)=0\right\}
$$


while for (3.7), that is, for the linear case

$$
\left[F_{0}-S\left(w^{0}, 0\right)\right]^{\perp}=\left[F_{0}-P_{K}\left(\Lambda^{-1} F_{0}\right)\right]^{\perp}=\left\{z \in H_{0}^{2}(\Omega):\left\langle F_{0}, z\right\rangle-A_{0}\left(w^{0}, z\right)=0\right\} .
$$

In $[9,10]$ the equality $(3.7)$, or $(3.4)$, is proven assuming an extra condition on the force $F_{0}$. It is a matter of verification that the arguments presented in $[9,10]$ to achieve this conclusion are still valid for the nonlinear case that is, when the orthogonal set (3.10) is replaced by the set (3.9), which includes the nonlinear term $a_{0}\left(G_{0}\left(w^{0}\right) ; w^{0}, z\right)$, resulting from the nonlinearity of the problem. So, in this section we state, whithout proof, the analogous of the propositions of $[9,10]$, which are fundamental for the proof of $(3.1)$, under an additional condition on $F_{0}$ and $w^{0}$ as established in Proposition 3.4.

We first remark that because $\left[F_{0}-S\left(w^{0}, 0\right)\right]^{\perp}$ is a closed set, then

$$
\overline{\left[F_{0}-S\left(w^{0}, 0\right)\right]^{\perp} \cap C_{K}\left(w^{0}\right)} \subset\left[F_{0}-S\left(w^{0}, 0\right)\right]^{\perp} \cap \overline{C_{K}\left(w^{0}\right)},
$$

so it is enough to prove that

$$
\left[F_{0}-S\left(w^{0}, 0\right)\right]^{\perp} \cap \overline{C_{K}\left(w^{0}\right)} \subset \overline{\left[F_{0}-S\left(w^{0}, 0\right)\right]^{\perp} \cap C_{K}\left(w^{0}\right)},
$$

in order to conclude that $K$ is a polyhedric set, at $w^{0}$, for $F_{0}-S\left(w^{0}, 0\right)$. The first result is the following:

Proposition 3.1. Let $w^{0} \in W(0)$, then, there exists a positive Radon measure $\mu$, defined by

$$
\int_{\Omega} \xi \mathrm{d} \mu=-\left\langle F_{0}-S\left(w^{0}, 0\right), \xi\right\rangle, \quad \forall \xi \in C_{0}^{\infty}(\Omega)
$$

whose support verifies

$$
\operatorname{supp} \mu \subset I=\left\{x \in \Omega: w^{0}(x)=0\right\},
$$

where $I$ is the set of points in $\Omega$ where $w^{0}$ touches the obstacle.

Proof. The proof is analogous to the proof of Theorem 1 in [10].

Before stating the next proposition, which gives a characterization of the set

$$
\overline{\left[F_{0}-S\left(w^{0}, 0\right)\right]^{\perp} \cap C_{K}\left(w^{0}\right)}
$$

we need to introduce the definition of admissible set ([10], Def. 2).

Definition 3.2. A compact set $L \subset \Omega$ is admissible if for every $\varphi \in H_{0}^{2}(\Omega)$, such that $\varphi=0 C_{2}$-q.e in $L$, then $\varphi \in H_{0}^{2}(\Omega \backslash L)$. The notation $C_{2}$-q.e in $L$, means that, the property $\varphi=0$ is satisfied in $L$, except in a subset $M$ of $L$, with $C_{2}$-capacity zero, that is [16]

$$
0=C_{2}(M)=\inf \left\{\int_{\Omega}|\Delta \varphi|^{2}: \quad \varphi \geq 1 \text { in } M, \quad 0 \leq \varphi \in C_{0}^{\infty}(\Omega)\right\} .
$$

Proposition 3.3. Let $\mu$ be the positive measure defined in Proposition 3.1 and assume supp $\mu$ is admissible. Then,

$$
\overline{\left[F_{0}-S\left(w^{0}, 0\right)\right]^{\perp} \cap C_{K}\left(w^{0}\right)}=\left\{\varphi \in H_{0}^{2}(\Omega \backslash \operatorname{supp} \mu): \varphi \geq 0 \text {, C } C_{2} \text {-q.e in } I\right\} \text {. }
$$


Proof. The proof is a straightforward adaptation of the proof of Theorem 2 in [10].

Finally the next proposition specifies the conditions on $\mu$, and consequently on $F_{0}$ and $w^{0}$, that imply the set $K$ is polyhedric in the sense of (3.1).

Proposition 3.4. If supp $\mu$ is admissible, then the set $K$ is polyhedric in the sense of (3.1).

Proof. As observed before and because of Proposition 3.3, it is enough to prove that

$$
\left[F_{0}-S\left(w^{0}, 0\right)\right]^{\perp} \cap \overline{C_{K}\left(w^{0}\right)} \subset\left\{\varphi \in H_{0}^{2}(\Omega \backslash \operatorname{supp} \mu): \varphi \geq 0, C_{2} \text {-q.e in } I\right\} .
$$

Let $\varphi \in\left[F_{0}-S\left(w^{0}, 0\right)\right]^{\perp} \cap \overline{C_{K}\left(w^{0}\right)}$. Then,

$$
\varphi=\lim _{n \rightarrow+\infty} \varphi_{n}, \quad \text { with } \quad \varphi_{n} \in C_{K}\left(w^{0}\right) .
$$

So by definition of $C_{K}\left(w^{0}\right)$, for each $n$,

$$
w^{0}+\lambda_{n} \varphi_{n} \geq 0, \quad \text { for some } \quad \lambda_{n}>0,
$$

so $\varphi_{n} \geq 0$ in $I$. Then $\left(\varphi_{n}\right)$ has a subsequence that converges to $\varphi C_{2}$-q.e [16], which implies also $\varphi \geq 0 C_{2}$-q.e in $I$. On the other hand, as $\varphi \in\left[F_{0}-S\left(w^{0}, 0\right)\right]^{\perp}$, and by the definition of $\mu$ we have

$$
0=\left\langle F_{0}-S\left(w^{0}, 0\right), \varphi\right\rangle=\int_{\Omega} \varphi \mathrm{d} \mu
$$

As $\varphi \geq 0 C_{2}$-q.e in $I$ and $\mu \geq 0$, we must have $\varphi=0 C_{2}$-q.e in $\operatorname{supp} \mu$. As $\operatorname{supp} \mu$ is an admissible set, then $\varphi \in H_{0}^{2}(\Omega \backslash \operatorname{supp} \mu)$ and so

$$
\varphi \in\left\{\varphi \in H_{0}^{2}(\Omega \backslash \operatorname{supp} \mu): \varphi \geq 0, C_{2} \text {-q.e in } I\right\} .
$$

As a consequence of this proposition the set $K^{*}$ in Theorem 2.7 is defined by

$$
K^{*}=\left\{w \in H_{0}^{2}(\Omega \backslash \operatorname{supp} \mu): w \geq 0, C_{2} \text {-q.e in } I\right\} .
$$

Remark 3.5. The set $K$ is also a polyhedric set at $\varphi^{0}$ for $F_{0}-V\left(\varphi^{0}, 0\right)$, that is

$$
\overline{\left[F_{0}-V\left(\varphi^{0}, 0\right)\right]^{\perp} \cap C_{K}\left(w^{0}\right)}=\left[F_{0}-V\left(\varphi^{0}, 0\right)\right]^{\perp} \cap \overline{C_{K}\left(w^{0}\right)}
$$

where $\varphi^{0}$ is the solution of (1.48) for $t=0$ and $V\left(\varphi^{0}, 0\right)=S\left(\varphi^{0}+\psi, 0\right)$ is defined by (1.49), that is, for any $z$ in $H_{0}^{2}(\Omega)$

$$
\left\langle V\left(\varphi^{0}, 0\right), z\right\rangle=A_{0}\left(\varphi^{0}+\psi, z\right)+a_{0}\left(G_{0}\left(\varphi^{0}+\psi\right) ; \varphi^{0}+\psi, z\right) .
$$

In fact, Propositions 3.3 and 3.4 are still valid with the measure $\mu$ of Proposition 3.1 defined by

$$
\int_{\Omega} \xi \mathrm{d} \mu=-\left\langle F_{0}-V\left(\varphi^{0}, 0\right), \xi\right\rangle, \quad \forall \xi \in C_{0}^{\infty}(\Omega)
$$




\section{The Semi-Derivative $D S\left(w^{0}, 0\right)$}

In this section we verify the second assumption of Theorem 2.7 , that is, $S$ is semi-differentiable at $\left(w^{0}, 0\right)$, and we compute this semi-derivative. In order to do the calculus of $D S\left(w^{0}, 0\right)$ we need some results about the operator $G_{t}$. The next proposition exhibits a continuity and a derivative result for $G_{t}$.

Proposition 4.1. Let $\phi=\left(\phi_{1}, \phi_{2}\right): \mathbb{R}^{+} \rightarrow H_{0}^{2}(\Omega) \times[0, \delta]$ such that

$$
\lim _{s \rightarrow 0^{+}} \frac{\phi_{1}(s)-w^{0}}{s}=w \text { in } H_{0}^{2}(\Omega) \text { strongly and } \lim _{s \rightarrow 0^{+}} \frac{\phi_{2}(s)}{s}=t \text { in }[0, \delta] .
$$

For each $s$ we define $\hat{\phi}_{1}(s)=\phi_{1}(s) \circ T_{\phi_{2}(s)}^{-1}$ and $G_{\phi_{2}(s)}\left(\hat{\phi}_{1}(s)\right)^{\phi_{2}(s)}=G_{\phi_{2}(s)}\left(\hat{\phi}_{1}(s)\right) \circ T_{\phi_{2}(s)}$. Then

$$
\lim _{s \rightarrow 0^{+}} G_{\phi_{2}(s)}\left(\hat{\phi}_{1}(s)\right)^{\phi_{2}(s)}=G_{0}\left(w^{0}\right) \text { in }\left[H_{0}^{1}(\Omega)\right]^{2} \text { strongly }
$$

and

$$
\lim _{s \rightarrow 0^{+}} \frac{G_{\phi_{2}(s)}\left(\hat{\phi}_{1}(s)\right)^{\phi_{2}(s)}-G_{0}\left(w^{0}\right)}{s}=h_{w, w^{0}} \text { in }\left[H_{0}^{1}(\Omega)\right]^{2} \text { strongly }
$$

where $h_{w, w^{0}} \in\left[H_{0}^{1}(\Omega)\right]^{2}$ is the solution of the equation

$$
B_{0}\left(h_{w, w^{0}}, \underline{v}\right)=-b^{*}\left(w^{0} ; w, \underline{v}\right)+t B_{1}\left(G_{0}\left(w^{0}\right), \underline{v}\right)-t b_{1}\left(w^{0}, \underline{v}\right), \quad \forall \underline{v} \in\left[H_{0}^{1}(\Omega)\right]^{2}
$$

with

$$
b^{*}\left(w^{0} ; w, \underline{v}\right)=h \int_{\Omega} C:\left(e(\underline{v}) \nabla w \nabla w^{0}\right) \mathrm{d} x .
$$

Proof. By (4.1) we first remark that

$$
\lim _{s \rightarrow 0^{+}} \phi_{1}(s)=w^{0} \text { in } H_{0}^{2}(\Omega) \text { strongly, and } \lim _{s \rightarrow 0^{+}} \phi_{2}(s)=0 \text { in }[0, \delta] .
$$

We now prove (4.2). From the definitions of $G_{\phi_{2}(s)}, G_{0}$ and using the decompositions of $B_{\phi_{2}(s)}, b_{\phi_{2}(s)}$, we have, for each $s$

$$
\begin{aligned}
& \left\{\begin{array}{l}
B_{0}\left(G_{\phi_{2}(s)}\left(\hat{\phi}_{1}(s)\right)^{\phi_{2}(s)}, \underline{v}\right)-\phi_{2}(s) B_{1}\left(G_{\phi_{2}(s)}\left(\hat{\phi}_{1}(s)\right)^{\phi_{2}(s)}, \underline{v}\right)= \\
-b_{0}\left(\phi_{1}(s), \underline{v}\right)+\phi_{2}(s) b_{1}\left(\phi_{1}(s), \underline{v}\right)+\mathcal{O}\left(\phi_{2}(s)^{2}\right), \quad \forall \underline{v} \in\left[H_{0}^{1}(\Omega)\right]^{2},
\end{array}\right. \\
& B_{0}\left(G_{0}\left(w^{0}\right), \underline{v}\right)=-b_{0}\left(w^{0}, \underline{v}\right), \quad \forall \underline{v} \in\left[H_{0}^{1}(\Omega)\right]^{2},
\end{aligned}
$$

where $\mathcal{O}\left(\phi_{2}(s)^{2}\right)$ is the term of order $\left(\phi_{2}(s)^{2}\right)$ defined in (1.43). Subtracting these equations we obtain

$$
\left\{\begin{array}{l}
B_{0}\left(G_{\phi_{2}(s)}\left(\hat{\phi}_{1}(s)\right)^{\phi_{2}(s)}-G_{0}\left(w^{0}\right), \underline{v}\right)=-b_{0}\left(\phi_{1}(s), \underline{v}\right)+b_{0}\left(w^{0}, \underline{v}\right) \\
+\phi_{2}(s) B_{1}\left(G_{\phi_{2}(s)}\left(\hat{\phi}_{1}(s)\right)^{\phi_{2}(s)}, \underline{v}\right)+\phi_{2}(s) b_{1}\left(\phi_{1}(s), \underline{v}\right)+\mathcal{O}\left(\phi_{2}(s)^{2}\right), \quad \forall \underline{v} \in\left[H_{0}^{1}(\Omega)\right]^{2} .
\end{array}\right.
$$

But from the definition of $b_{0}$ we have

$$
\left\{\begin{array}{l}
b_{0}\left(\phi_{1}(s), \underline{v}\right)-b_{0}\left(w^{0}, \underline{v}\right)=\frac{h}{2} \int_{\Omega} C:\left\{e(\underline{v})\left(\nabla \phi_{1}(s) \nabla \phi_{1}(s)-\nabla w^{0} \nabla w^{0}\right)\right\}= \\
\frac{h}{2} \int_{\Omega} C:\left\{e(\underline{v})\left(\nabla \phi_{1}(s)+\nabla w^{0}\right)\left(\nabla \phi_{1}(s)-\nabla w^{0}\right)\right\}
\end{array}\right.
$$


Now, because the norm $\|e(\underline{v})\|_{\left[L^{2}(\Omega)\right]^{4}}$ is a norm equivalent to $\|\underline{v}\|_{\left[H_{0}^{1}(\Omega)\right]^{2}}$ in $\left[H_{0}^{1}(\Omega)\right]^{2}$, we have

$$
\left|b_{0}\left(\phi_{1}(s), \underline{v}\right)-b_{0}\left(w^{0}, \underline{v}\right)\right| \leq c_{0}\|\underline{v}\|_{\left[H_{0}^{1}(\Omega)\right]^{2}}\left\|\nabla\left(\phi_{1}(s)+w^{0}\right)\right\|_{\left[L^{4}(\Omega)\right]^{2}}\left\|\nabla\left(\phi_{1}(s)-w^{0}\right)\right\|_{\left[L^{4}(\Omega)\right]^{2}},
$$

where $c_{0}$ is a constant independent of $s$. Also from the definition of $b_{1}$

$$
\left|\phi_{2}(s) b_{1}\left(\phi_{1}(s), \underline{v}\right)\right| \leq c_{1}\left|\phi_{2}(s)\right|\|\underline{v}\|_{\left[H_{0}^{1}(\Omega)\right]^{2}}\left\|\nabla \phi_{1}(s)\right\|_{\left[L^{4}(\Omega)\right]^{2}}^{2}
$$

where $c_{1}$ is a constant independent of $s$. If we choose now in (4.9)

$$
\underline{v}=G_{\phi_{2}(s)}\left(\hat{\phi}_{1}(s)\right)^{\phi_{2}(s)}-G_{0}\left(w^{0}\right),
$$

and using the ellipticity of $B_{0}$, the continuity of $B_{1}$ and estimates $(4.11,4.12)$, we obtain from $(4.9)$

$$
\left\{\begin{array}{l}
\left\|G_{\phi_{2}(s)}\left(\hat{\phi}_{1}(s)\right)^{\phi_{2}(s)}-G_{0}\left(w^{0}\right)\right\|_{\left[H_{0}^{1}(\Omega)\right]^{2}} \leq \\
c_{2}\left|\phi_{2}(s)\right|\left\|G_{\phi_{2}(s)}\left(\hat{\phi}_{1}(s)\right)^{\phi_{2}(s)}\right\|_{\left[H_{0}^{1}(\Omega)\right]^{2}}+c_{3}\left|\phi_{2}(s)\right|\left\|\nabla \phi_{1}(s)\right\|_{\left[L^{4}(\Omega)\right]^{2}}^{2} \\
+c_{4}\left\|\nabla \phi_{1}(s)-\nabla w^{0}\right\|_{\left[L^{4}(\Omega)\right]^{2}}\left\|\nabla \phi_{1}(s)+\nabla w^{0}\right\|_{\left[L^{4}(\Omega)\right]^{2}}+\mathcal{O}\left(\phi_{2}(s)^{2}\right)
\end{array}\right.
$$

where $c_{2}, c_{3}$ and $c_{4}$ are constants independent of $s$. But $\left\|G_{\phi_{2}(s)}\left(\hat{\phi}_{1}(s)\right)^{\phi_{2}(s)}\right\|_{\left[H_{0}^{1}(\Omega)\right]^{2}}$ is majorated by a constant independent of $s$. In fact, from the definition of $G_{\phi_{2}(s)}\left(\hat{\phi}_{1}(s)\right)$ we obtain, for each $s$,

$$
B_{\phi_{2}(s)}\left(G_{\phi_{2}(s)}\left(\hat{\phi}_{1}(s)\right), \underline{v}_{\phi_{2}(s)}\right)=-b_{\phi_{2}(s)}\left(\hat{\phi}_{1}(s), \underline{v}_{\phi_{2}(s)}\right), \quad \forall \underline{v}_{\phi_{2}(s)} \in\left[H_{0}^{1}\left(\Omega_{\phi_{2}(s)}\right)\right]^{2},
$$

and choosing $\underline{v}_{\phi_{2}(s)}=G_{\phi_{2}(s)}\left(\hat{\phi}_{1}(s)\right)$, using the ellipticity of $B_{\phi_{2}(s)}$, the continuity of $b_{\phi_{2}(s)}$ and the relations between the norms of the spaces $\left[H_{0}^{1}\left(\Omega_{\phi_{2}(s)}\right)\right]^{2},\left[H_{0}^{1}(\Omega)\right]^{2}$ and $\left[L^{4}\left(\Omega_{\phi_{2}(s)}\right)\right]^{2},\left[L^{4}(\Omega)\right]^{2}$ we deduce

$$
\left\|G_{\phi_{2}(s)}\left(\hat{\phi}_{1}(s)\right)^{\phi_{2}(s)}\right\|_{\left[H_{0}^{1}(\Omega)\right]^{2}} \leq c_{5}\left\|\nabla \phi_{1}(s)\right\|_{\left[L^{4}(\Omega)\right]^{2}}^{2},
$$

with $c_{5}$ a constant independent of $s$. As $\phi_{1}(s)$ converges to $w^{0}$ in $H_{0}^{2}(\Omega)$, and since $H_{0}^{2}(\Omega)$ is compactly embedded in $W^{1,4}(\Omega)$, also $\nabla \phi_{1}(s)$ converges to $\nabla w^{0}$ in $\left[L^{4}(\Omega)\right]^{2}$. So $\nabla \phi_{1}(s)$ is a limited sequence in $\left[L^{4}(\Omega)\right]^{2}$ and from (4.16) we have

$$
\left\|G_{\phi_{2}(s)}\left(\hat{\phi}_{1}(s)\right)^{\phi_{2}(s)}\right\|_{\left[H_{0}^{1}(\Omega)\right]^{2}} \leq c_{6},
$$

with $c_{6}$ a constant independent of $s$. Finally, taking the limit as $s \rightarrow 0^{+}$, in the second member of (4.14) we have $(4.2)$, since $\lim _{s \rightarrow 0^{+}} \mathcal{O}\left(\phi_{2}(s)^{2}\right)=0$, because of $(4.17,1.43)$ and $(4.6)$.

The proof of (4.3) follows the same reasoning of (4.2). In fact, if we divise by $s$, the equations $(4.7,4.8)$, and subtract, we find

$$
\left\{\begin{array}{l}
B_{0}\left(\frac{1}{s}\left(G_{\phi_{2}(s)}\left(\hat{\phi}_{1}(s)\right)^{\phi_{2}(s)}-G_{0}\left(w^{0}\right)\right), \underline{v}\right)=-\frac{1}{s} b_{0}\left(\phi_{1}(s), \underline{v}\right)+\frac{1}{s} b_{0}\left(w^{0}, \underline{v}\right) \\
+\frac{\phi_{2}(s)}{s} B_{1}\left(G_{\phi_{2}(s)}\left(\hat{\phi}_{1}(s)\right)^{\phi_{2}(s)}, \underline{v}\right)+\frac{\phi_{2}(s)}{s} b_{1}\left(\phi_{1}(s), \underline{v}\right)+\frac{1}{s} \mathcal{O}\left(\phi_{2}(s)^{2}\right), \quad \forall \underline{v} \in\left[H_{0}^{1}(\Omega)\right]^{2} .
\end{array}\right.
$$

We now analyse the limits of the terms in the second member of (4.18). From (4.10) and because

$$
\left\{\begin{array}{l}
\lim _{s \rightarrow 0^{+}} \nabla \phi_{1}(s)=\nabla w^{0} \text { in }\left[L^{4}(\Omega)\right]^{2} \text { strongly } \\
\lim _{s \rightarrow 0^{+}} \frac{\nabla \phi_{1}(s)-\nabla w^{0}}{s}=\nabla w \text { in }\left[L^{4}(\Omega)\right]^{2} \text { strongly }
\end{array}\right.
$$


then

$$
\lim _{s \rightarrow 0^{+}}\left[\frac{1}{s} b_{0}\left(\phi_{1}(s), \underline{v}\right)-\frac{1}{s} b_{0}\left(w^{0}, \underline{v}\right)\right]=h \int_{\Omega} C:\left(e(\underline{v}) \nabla w^{0} \nabla w\right)=b^{*}\left(w^{0}, w, \underline{v}\right) .
$$

On the other hand, equations $(4.1,4.2)$ and the continuity of $B_{1}$ give

$$
\lim _{s \rightarrow 0^{+}} \frac{\phi_{2}(s)}{s} B_{1}\left(G_{\phi_{2}(s)}\left(\hat{\phi}_{1}(s)\right)^{\phi_{2}(s)}, \underline{v}\right)=t B_{1}\left(G_{0}\left(w^{0}\right), \underline{v}\right) .
$$

Using also the definition of $b_{1}$, equations (4.1) and (4.6) we obtain

$$
\lim _{s \rightarrow 0^{+}} \frac{\phi_{2}(s)}{s} b_{1}\left(\phi_{1}(s), \underline{v}\right)=t b_{1}\left(w^{0}, \underline{v}\right) .
$$

Observing finally that, from $(4.17,1.43)$ and $(4.6), \lim _{s \rightarrow 0^{+}} \frac{1}{s} \mathcal{O}\left(\phi_{2}(s)^{2}\right)=0$, the statement (4.3) follows from (4.18), because of (4.20-4.22).

We have now all the results to compute $D S\left(w^{0}, 0\right)(w, t)$.

Proposition 4.2. Let $\phi=\left(\phi_{1}, \phi_{2}\right): \mathbb{R}^{+} \rightarrow H_{0}^{2}(\Omega) \times[0, \delta]$ satisfying (4.1). Then the semi-derivative of $S$ $D S\left(w^{0}, 0\right)(w, t)$ exists and its definition, for each $z \in H_{0}^{2}(\Omega)$, is the following:

$$
\left\{\begin{array}{l}
D S\left(w^{0}, 0\right)(w, t)(z)=\lim _{s \rightarrow 0^{+}}\left\langle\frac{S\left(\phi_{1}(s), \phi_{2}(s)\right)-S\left(w^{0}, 0\right)}{s}, z\right\rangle= \\
A_{0}(w, z)-t A_{1}\left(w^{0}, z\right)-t F_{1}(z)-t a_{1}\left(G_{0}\left(w^{0}\right) ; w^{0}, z\right)+t a^{*}\left(w^{0} ; w, z\right)
\end{array}\right.
$$

where

$$
a^{*}\left(w^{0} ; w, z\right)=h \int_{\Omega} C:\left\{\left[e\left(h_{w, w^{0}}\right) \nabla w^{0}+e\left(G_{0}\left(w^{0}\right)\right) \nabla w\right] \nabla z+\frac{3}{2} \nabla w^{0} \nabla w^{0} \nabla w \nabla z\right\} \mathrm{d} x
$$

and $h_{w, w^{0}}$ is the solution of equation (4.4).

Proof. For any $z \in H_{0}^{2}(\Omega)$ we have, due to the definition of $S$ that

$$
\left\{\begin{array}{l}
\left\langle\frac{S\left(\phi_{1}(s), \phi_{2}(s)\right)-S\left(w^{0}, 0\right)}{s}, z\right\rangle=\frac{A_{0}\left(\phi_{1}(s), z\right)-A_{0}\left(w^{0}, z\right)}{s}-\frac{\phi_{2}(s)}{s} A_{1}\left(\phi_{1}(s), z\right) \\
+\frac{1}{s}\left[a_{0}\left(G_{\phi_{2}(s)}\left(\hat{\phi}_{1}(s)\right)^{\phi_{2}(s)} ; \phi_{1}(s), z\right)-a_{0}\left(G_{0}\left(w^{0}\right) ; w^{0}, z\right)\right] \\
-\frac{\phi_{2}(s)}{s} F_{1}(z)-\frac{\phi_{2}(s)}{s} a_{1}\left(G_{\phi_{2}(s)}\left(\hat{\phi}_{1}(s)\right)^{\phi_{2}(s)} ; \phi_{1}(s), z\right)+\frac{1}{s} \mathcal{O}\left(\phi_{2}(s)^{2}\right),
\end{array}\right.
$$

where $\mathcal{O}\left(\phi_{2}(s)^{2}\right)$ is defined in (1.41).

We compute now the limit of each term in the second member in (4.25). Using the definitions of $A_{0}$ and $A_{1}$ we directly obtain

$$
\begin{aligned}
& \lim _{s \rightarrow 0^{+}} \frac{A_{0}\left(\phi_{1}(s), z\right)-A_{0}\left(w^{0}, z\right)}{s}=A_{0}\left(\lim _{s \rightarrow 0^{+}} \frac{\phi_{1}(s)-w^{0}}{s}, z\right)=A_{0}(w, z) \\
& \lim _{s \rightarrow 0^{+}} \frac{\phi_{2}(s)}{s} A_{1}\left(\phi_{1}(s), z\right)=t A_{1}(w, z) .
\end{aligned}
$$

Also it is clear that

$$
\lim _{s \rightarrow 0^{+}} \frac{\phi_{2}(s)}{s} F_{1}(z)=t F_{1}(z) \text { and } \lim _{s \rightarrow 0^{+}} \frac{1}{s} \mathcal{O}\left(\phi_{2}(s)^{2}\right)=0 .
$$


The second limit in $(4.27)$ is a consequence of $(4.17,1.41)$ and $(4.6)$.

From $(4.2,4.6)$ and the continuity of $a_{1}$

$$
\lim _{s \rightarrow 0^{+}} \frac{\phi_{2}(s)}{s} a_{1}\left(G_{\phi_{2}(s)}\left(\hat{\phi}_{1}(s)\right)^{\phi_{2}(s)} ; \phi_{1}(s), z\right)=t a_{1}\left(G_{0}\left(w^{0}\right) ; w^{0}, z\right) .
$$

Finally using the definition of $a_{0}$

$$
\left\{\begin{array}{l}
\lim _{s \rightarrow 0^{+}} \frac{1}{s}\left[a_{0}\left(G_{\phi_{2}(s)}\left(\hat{\phi}_{1}(s)\right)^{\phi_{2}(s)} ; \phi_{1}(s), z\right)-a_{0}\left(G_{0}\left(w^{0}\right) ; w^{0}, z\right)\right]= \\
\lim _{s \rightarrow 0^{+}} \frac{h}{s} \int_{\Omega} C:\left[e\left(G_{\phi_{2}(s)}\left(\hat{\phi}_{1}(s)\right)^{\phi_{2}(s)}\right) \nabla \phi_{1}(s)-e\left(G_{0}\left(w^{0}\right)\right) \nabla w^{0}\right] \nabla z \mathrm{~d} x+ \\
\lim _{s \rightarrow 0^{+}} \frac{h}{2 s} \int_{\Omega} C:\left[\nabla \phi_{1}(s) \nabla \phi_{1}(s) \nabla \phi_{1}(s)-\nabla w^{0} \nabla w^{0} \nabla w^{0}\right] \nabla z \mathrm{~d} x
\end{array}\right.
$$

But

$$
\left\{\begin{array}{c}
\lim _{s \rightarrow 0^{+}} \frac{h}{s} \int_{\Omega} C:\left[e\left(G_{\phi_{2}(s)}\left(\hat{\phi}_{1}(s)\right)^{\phi_{2}(s)}\right) \nabla \phi_{1}(s)-e\left(G_{0}\left(w^{0}\right)\right) \nabla w^{0}\right] \nabla z \mathrm{~d} x= \\
\lim _{s \rightarrow 0^{+}} h \int_{\Omega} C:\left[e\left(\frac{G_{\phi_{2}(s)}\left(\hat{\phi}_{1}(s)\right)^{\phi_{2}(s)}-G_{0}\left(w^{0}\right)}{s}\right) \nabla \phi_{1}(s)+\right. \\
\left.e\left(G_{0}\left(w^{0}\right)\right) \frac{\nabla \phi_{1}(s)-\nabla w^{0}}{s}\right] \nabla z \mathrm{~d} x= \\
h \int_{\Omega} C:\left[e\left(h_{w, w^{0}}\right) \nabla w^{0}+e\left(G_{0}\left(w^{0}\right)\right) \nabla w\right] \nabla z \mathrm{~d} x
\end{array}\right.
$$

and

$$
\left\{\begin{array}{l}
\lim _{s \rightarrow 0^{+}} \frac{h}{2 s} \int_{\Omega} C:\left[\nabla \phi_{1}(s) \nabla \phi_{1}(s) \nabla \phi_{1}(s)-\nabla w^{0} \nabla w^{0} \nabla w^{0}\right] \nabla z \mathrm{~d} x= \\
\lim _{s \rightarrow 0^{+}} \frac{h}{2} \int_{\Omega} C:\left[\left(\nabla \phi_{1}(s) \nabla \phi_{1}(s)+\nabla \phi_{1}(s) \nabla w^{0}+\nabla w^{0} \nabla w^{0}\right) \frac{\nabla \phi_{1}(s)-\nabla w^{0}}{s}\right] \nabla z \mathrm{~d} x= \\
\frac{3 h}{2} \int_{\Omega} C:\left[\nabla w^{0} \nabla w^{0} \nabla w\right] \nabla z \mathrm{~d} x .
\end{array}\right.
$$

Remark 4.3. The mapping $V(z, t)$ defined in (1.49) is also semi-differentiable at $\left(\varphi^{0}, 0\right)$, with $\varphi^{0}$ a solution of (1.48), for $t=0$. In fact, as $V(z, t)=S(z+\psi, t)$, with the obstacle $\psi$ smooth enough, and because $S$ is semi-differentiable at $\left(\varphi^{0}+\psi, 0\right)$, a simple calculus shows that the semi-derivative of $V$ at $\left(\varphi^{0}, 0\right)$ is defined by

$$
D V\left(\varphi^{0}, 0\right)(\varphi, t)=D S\left(\varphi^{0}+\psi, 0\right)\left(\varphi+t(\nabla \psi)^{T} . \theta, t\right), \quad \forall(\varphi, t) \in H_{0}^{2}(\Omega) \times[0, \delta] .
$$

So, taking into account the Remark 3.5, we conclude that Theorem 2.7 applies to problem (1.48), with $\psi \neq 0$. That is, the proto-derivative of the multifunction mapping

$$
\Phi(t)=\left\{\varphi^{t}: \varphi^{t} \text { solution of problem (1.48) }\right\}
$$

exists at $t=0$, and is defined by

$$
D \Phi(0)\left(\varphi^{0}\right)(t)=\left\{\varphi \in K^{*}: \quad\left\langle-D V\left(\varphi^{0}, 0\right)(\varphi, t), z-w\right\rangle \leq 0, \quad \forall z \in K^{*}\right\},
$$


with

$$
K^{*}=\overline{\left[F_{0}-V\left(\varphi^{0}, 0\right)\right]^{\perp} \cap \cup_{\lambda>0} \lambda\left(K-w^{0}\right)} .
$$

Consequently, the multifunction mapping

$$
W(t)=\Phi(t)+\psi^{t}=\left\{w^{t}=\varphi^{t}+\psi^{t}\right\},
$$

whose elements are solutions of problem (1.45), is proto-differentiable at $t=0$, with proto-derivative equal to

$$
D W(0)\left(w^{0}\right)(t)=D \Phi(0)\left(\varphi^{0}\right)(t)+(\nabla \psi)^{T} \cdot \theta
$$

for any $t \in[0, \delta]$. This proto-derivative quantifies the shape sensitivity of the solution of problem (1.45) at $t=0$.

\section{Sensitivity of the linear obstacle Plate PROBlem}

The shape sensitivity analysis of the linear obstacle plate problem is done in $[9,10]$. This analysis is based on the differentiability of the projection operator $[4,8]$ and on a sensitivity result for an abstract variational inequality [13]. In this section we show that this sensitivity result can also be obtained using the proto-derivative. That is, Theorem 2.7 also applies to the linear obstacle plate problem, and gives exactly the same result as in $[9,10]$.

In fact the linear obstacle plate problem is characterized by the following variational inequality obtained from the nonlinear system $(1.13,1.14)$, by neglecting the nonlinear terms,

$$
\left\{\begin{array}{l}
\text { Find } w_{t} \in K_{t}: \\
A_{t}\left(w_{t}, z_{t}-w_{t}\right) \geq F_{t}\left(z_{t}-w_{t}\right), \quad \forall z_{t} \in K_{t}
\end{array}\right.
$$

This problem can be transported to the fixed domain $\Omega$, as in the nonlinear case, and for the obstacle $\psi$ equal to zero (5.1) becomes

$$
\left\{\begin{array}{l}
\text { Find } w^{t} \in K=\left\{z \in H_{0}^{2}(\Omega): z \geq 0 \quad \text { in } \quad \Omega\right\}: \\
\left\langle F_{0}-S\left(w^{t}, t\right), z-w^{t}\right\rangle \leq 0, \quad \forall z \in K
\end{array}\right.
$$

with $S$ defined by

$$
\left\{\begin{array}{l}
\langle S(w, t), z\rangle=A_{0}(w, z)-t A_{1}(w, z)-t F_{1}(z)+\mathcal{O}\left(t^{2}\right), \\
\forall(w, t) \in H_{0}^{2}(\Omega) \times[0, \delta], \quad \forall z \in H_{0}^{2}(\Omega) .
\end{array}\right.
$$

A direct application of the results of $[8-10,13]$ enable to conclude that the unique solution of $(5.2)$ is rightdifferentiable at $t=0$, with the derivative $\dot{w}(0)$ (which is the material derivative) verifying

$$
\lim _{t \rightarrow 0^{+}} \frac{w^{t}-w^{0}}{t}=\dot{w}(0) \quad \text { in } \quad H_{0}^{2}(\Omega) \text { strongly } \quad \text { and } \quad \dot{w}(0)=P_{K^{*}}\left(\Lambda^{-1}\left(F_{1}+A_{1}\left(w^{0}, .\right)\right)\right) .
$$

In (5.4) the function $w^{0}$ is the unique solution of (5.2) for $t=0$, and is equal to $P_{K}\left(\Lambda^{-1} F_{0}\right)$, where $\Lambda$ is the isometry (defined in Sect. 3) between $H_{0}^{2}(\Omega)$ and its dual and $P_{K}$ the $A_{0}$-projection on the set $K$, that is,

$$
\left\{\begin{array}{l}
w^{0}=P_{K}\left(\Lambda^{-1} F_{0}\right) \in K \\
A_{0}\left(w^{0}, z-w^{0}\right) \geq\left\langle F_{0}, z-w^{0}\right\rangle, \quad \forall z \in K
\end{array}\right.
$$


Moreover the set $K^{*}$ is defined by

$$
K^{*}=\left[F_{0}-A_{0}\left(w^{0}, .\right)\right]^{\perp} \cap \overline{C_{K}\left(w^{0}\right)}
$$

and $P_{K^{*}}$ denotes the $A_{0}$-projection on the set $K^{*}$, that is

$$
\left\{\begin{array}{l}
\dot{w}(0)=P_{K^{*}}\left(\Lambda^{-1}\left(F_{1}+A_{1}\left(w^{0}, .\right)\right)\right) \in K^{*} \\
A_{0}(\dot{w}(0), z-\dot{w}(0)) \geq\left\langle F_{1}+A_{1}\left(w^{0}, .\right), z-\dot{w}(0)\right\rangle, \quad \forall z \in K^{*} .
\end{array}\right.
$$

The next result shows that for the linear obstacle plate problem (5.2) the results of Theorem 2.7 recover the sensitivity result (5.4).

Proposition 5.1. Let $W(t)$ be the function that assigns to each the unique solution of problem (5.2) and $w^{0}=W(0)$. Then, the proto-derivative $D W(0)\left(w^{0}\right)$ exists, coincides with the semi-derivative $D W(0)$ and is defined by

$$
D W(0)\left(w^{0}\right)(t)=t P_{K^{*}}\left(\Lambda^{-1}\left(F_{1}+A_{1}\left(w^{0}, .\right)\right)\right)
$$

for each $t \in[0, \delta]$. In addition the semi-derivative satisfies

$$
D W(0)(t)=t \lim _{s \rightarrow 0^{+}} \frac{w^{s}-w^{0}}{s}, \quad \text { in } H_{0}^{2}(\Omega) \text { strongly }
$$

and consequently $(5.8,5.9)$ give (5.4).

Proof. By (4.23), the semi-derivative of function $S$, defined in $(5.3)$, at $\left(w^{0}, 0\right)$ is

$$
D S\left(w^{0}, 0\right)(w, t)(z)=A_{0}(w, z)-t F_{1}(z)-t A_{1}\left(w^{0}, z\right) .
$$

On the other hand, as proved in $[9,10], K$ is a convex set, that is polyhedric at $w^{0}$, for $F_{0}-S\left(w^{0}, 0\right)$, and

$$
\overline{\left[F_{0}-S\left(w^{0}, 0\right)\right]^{\perp} \cap \cup_{\lambda{ }_{0}} \lambda\left(K-w^{0}\right)}=\overline{\left[F_{0}-S\left(w^{0}, 0\right)\right]^{\perp} \cap C_{K}\left(w^{0}\right)}=\left[F_{0}-S\left(w^{0}, 0\right)\right]^{\perp} \cap \overline{C_{K}\left(w^{0}\right)}=K^{*} .
$$

Thus the two assumptions of Theorem 2.7 are fulfilled and therefore the proto-derivative of the single-valued function $W(t)$, solution of (5.2), at $t=0$ is defined by

$$
D W(0)\left(w^{0}\right)(t)=\left\{w \in K^{*}: A_{0}(w, z-w) \geq\left\langle t F_{1}+t A_{1}\left(w^{0}, .\right), z-w\right\rangle, \forall z \in K^{*}\right\} .
$$

But this means, by definition of $P_{K^{*}}$, that each element $w$ of $D W(0)\left(w^{0}\right)(t)$ is the projection of $\Lambda^{-1}\left(t F_{1}+\right.$ $\left.t A_{1}\left(w^{0},.\right)\right)$ on the closed subspace $K^{*}$. So the set $D W(0)\left(w^{0}\right)(t)$ has only one element, that is

$$
D W(0)\left(w^{0}\right)(t)=P_{K^{*}}\left(\Lambda^{-1}\left(t F_{1}+t A_{1}\left(w^{0}, .\right)\right)\right)=t P_{K^{*}}\left(\Lambda^{-1}\left(F_{1}+A_{1}\left(w^{0}, .\right)\right)\right)
$$

because $P_{K^{*}}$ is linear, and $t$ is a scalar. Finally, by the Proposition $2.5(\mathrm{i})$, the proto-derivative $D W(0)\left(w^{0}\right)$ is equal to the semi-derivative $D W(0)$ and it is also simple to deduce, using the definition of semi-derivative, that $(5.9)$ is verified, that is, $D W(0)(t)=t \dot{w}(0)$, where $\dot{w}(0)$ is the material derivative defined in (5.4). So

$$
t \dot{w}(0)=D W(0)(t)=D W(0)\left(w^{0}\right)(t)=t P_{K^{*}}\left(\Lambda^{-1}\left(F_{1}+A_{1}\left(w^{0}, .\right)\right)\right)
$$

which is precisely the result (5.4) established by $[9,10]$. 


\section{Conclusion}

In this paper we prove two properties that guarantee the existence of the proto-derivative of the solution multifunction mapping associated to the variational inequality defining the nonlinear obstacle plate problem. This proto-derivative characterizes the shape sensitivity of the solution mapping. As proved in an abstract setting by [6], these two properties concern the set of constraints defined by the obstacle, which must be a polyhedric set, in the sense of Definition 2.1, and the operator defining the variational inequality, that must be semi-differentiable in the sense of Definition 2.2. Using a straightforward adaptation of the proof of $[9,10]$ for the linear case, we show that the set of constraints is polyhedric, under an additional condition imposed on the force acting on the plate, as explained in Proposition 3.4. The semi-differentiability of the operator is proven in Section 4, and relies on the continuity, ellipticity and differentiability properties of the operators (1.18-1.22), despite the nonlinearity of the problem. We intend to apply this methodology to analyse the sensitivity of other problems, as shell problems, and to develop numerical methods to solve the corresponding finite element approximations $[2,3]$.

\section{REFERENCES}

[1] H. Brézis, Equations et inéquations nonlinéaires dans les espaces vectoriels en dualité. Ann. Inst. Fourier (Grenoble 18 (1968) $115-175$.

[2] J. Haslinger and P. Neittaanmäki, Finite element approximation for optimal shape design, theory and applications. Wiley, Chichester (1988).

[3] J. Haslinger, M. Miettinen and P. Panagiotopoulos, Finite element method for hemivariational inequalities. Theory, methods and applications. Kluwer Academic Publishers (1999).

[4] A. Haraux, How to differentiate the projection on a convex set in Hilbert space. Some applications to variational inequalities. J. Math. Soc. Japan 29 (1977) 615-631.

[5] N. Kikuchi and J.T. Oden, Contact problems in elasticity: A study of variational inequalities and finite element methods. SIAM (1988).

[6] A.B. Levy, Sensitivity of solutions to variational inequalities on Banach Spaces. SIAM J. Control Optim. 38 (1999) 50-60.

[7] A.B. Levy and R.T. Rockafeller, Sensitivity analysis of solutions to generalized equations. Trans. Amer. Math. Soc. 345 (1994) 661-671.

[8] F. Mignot, Contrôle dans les inéquations variationnelles elliptiques. J. Funct. Anal. 22 (1976) 130-185.

[9] M. Rao and J. Sokolowski, Sensitivity analysis of Kirchhoff plate with obstacle, Rapports de Recherche, 771. INRIA-France (1987).

[10] M. Rao and J. Sokolowski, Sensitivity analysis of unilateral problems in $H_{0}^{2}(\Omega)$ and applications. Numer. Funct. Anal. Optim. 14 (1993) 125-143.

[11] R.T. Rockafeller, Proto-differentiability of set-valued mappings and its applications in Optimization. Ann. Inst. H. Poincaré Anal. Non Linéaire 6 (1989) 449-482.

[12] A. Shapiro, On concepts of directional differentiability. J. Optim. Theory Appl. 66 (1990) 477-487.

[13] J. Sokolowski and J.-P. Zolesio, Shape sensitivity analysis of unilateral problems. SIAM J. Math. Anal. 18 (1987) $1416-1437$.

[14] J. Sokolowski and J.-P. Zolesio, Shape design sensitivity analysis of plates and plane elastic solids under unilateral constraints. J. Optim. Theory Appl. 54 (1987) 361-382.

[15] J. Sokolowski and J.-P. Zolesio, Introduction to shape optimization - shape sensitivity analysis. Springer-Verlag, Springer Ser. Comput. Math. 16 (1992).

[16] P.W. Ziemer, Weakly differentiable functions. Springer-Verlag, New York (1989). 